\title{
Photoluminescence control by hyperbolic metamaterials and metasurfaces: a review
}

\author{
Leonid Yu. Beliaev ${ }^{1}$, Osamu Takayama ${ }^{1}$, Pavel N. Melentiev ${ }^{2,3}$ and \\ Andrei V. Lavrinenko ${ }^{1 *}$
}

\begin{abstract}
Photoluminescence including fluorescence plays a great role in a wide variety of applications from biomedical sensing and imaging to optoelectronics. Therefore, the enhancement and control of photoluminescence has immense impact on both fundamental scientific research and aforementioned applications. Among various nanophotonic schemes and nanostructures to enhance the photoluminescence, we focus on a certain type of nanostructures, hyperbolic metamaterials (HMMs). HMMs are highly anisotropic metamaterials, which produce intense localized electric fields. Therefore, HMMs naturally boost photoluminescence from dye molecules, quantum dots, nitrogen-vacancy centers in diamonds, perovskites and transition metal dichalcogenides. We provide an overview of various configurations of HMMs, including metal-dielectric multilayers, trenches, metallic nanowires, and cavity structures fabricated with the use of noble metals, transparent conductive oxides, and refractory metals as plasmonic elements. We also discuss lasing action realized with HMMs.
\end{abstract}

Keywords: fluorescence; metamaterials; metasurfaces; Purcell effect; nanophotonics; hyperbolic metamaterials

Beliaev LY, Takayama O, Melentiev PN, Lavrinenko AV. Photoluminescence control by hyperbolic metamaterials and metasurfaces: a review. Opto-Electron Adv 4, 210031 (2021).

\section{Introduction}

Photoluminescence (PL), emission of light from materials, have been widely used for numerous applications ranging from imaging of biological specimen to lasers. Light emitters can take various forms in different material, e.g., fluorescent molecules, quantum dots (QDs), or nitrogen-vacancy centers in diamond ${ }^{1}$ (NVC). The applications include single molecular detection ${ }^{2}$, biosensing $^{3-7}$ and imaging ${ }^{8-10}$, where a fluorophore as a label is attached to analyte molecules in order to image them or detect their concentration linked with the intensity of emitted fluorescence signals. Detection schemes with fluorescence markers attached to analytes are routinely used in clinical diagnosis. Moreover, for optoelectronic emitting devices it is desirable to enhance their PL emission to improve efficiency of light sources, such as LEDs, lasers, and single-photon sources, since PL is the essential part of their emitting schemes ${ }^{11,12}$. This is the main motivation behind many of the recent developments in engineering spontaneous emission using nanoscale structures. Up to present, there have been numerous studies to boost PL by plasmonic, e.g. metal, nanostructures ${ }^{3}$. For instance, a silver thin film on a glass substrate supports highly localized electric field by mean of a surface plasmon polariton, which strengthens the fluorescence signals of the fluorophores by more than $300 \%{ }^{13}$. Plasmonic nanoantennas tightly focus light within the structure, and thus are routinely used for the

1DTU Fotonik-Department of Photonics Engineering, Technical University of Denmark, Ørsteds Plads 343, DK-2800 Kgs. Lyngby, Denmark; ${ }^{2}$ Institute of Spectroscopy RAS, Moscow 108840, Russia; ${ }^{3}$ Higher School of Economics, National Research University, Moscow 101000 , Russia. "Correspondence: Lavrinenko AV, E-mail: alav@fotonik.dtu.dk

Received: 5 March 2021; Accepted: 29 May 2021; Published: 25 August 2021

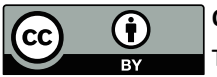

Open Access This article is licensed under a Creative Commons Attribution 4.0 International License.

To view a copy of this license, visit http://creativecommons.org/licenses/by/4.0/.

(C) The Author(s) 2021. Published by Institute of Optics and Electronics, Chinese Academy of Sciences. 
enhancement of fluorescent signals ${ }^{2,14,15}$. Interested readers may find review articles on plasmonic nanostructures including plasmonic nanoparticles and gratings for fluorescence enhancement elsewhere ${ }^{16,17}$. Another current tendency is to localize fields by Mie-resonances in high-index dielectric nanostructures, such as multilayer stacks $^{18-20}$, 1D gratings ${ }^{21-27}, 2 \mathrm{D}$ photonic crystal structures $^{28}$, as well as, metasurfaces made of dielectric particles $^{29-31}$.

In this review, we focus on the enhancement of PL by a class of metamaterials, named hyperbolic metamaterials (HMMs), which are also referred to as an indefinite media, and hyperbolic metasurfaces (HMSs) ${ }^{32-42}$. Hyperbolic materials are known for the extreme anisotropy and unique dispersion bands in the wavevector space ( $\mathrm{k}$ space) generated by the principal permittivity tensor components being of opposite signs. These bands take the shape of a hyperboloid as opposed to spherical or ellipsoid shapes of dispersion contours of dielectric materials. HMMs feature highly localized bulk waves that propagate within the structures which can be used, for example, for sensing ${ }^{43-45}$, sub-diffraction imaging ${ }^{46}$, and directional emission of light by quantum emitters ${ }^{47,48}$. The underlying features used for the control of PL by HMMs are large accepted wavevectors (high-k case), highly localized fields and large optical density of states, leading to enhanced light-matter interactions (increase in the Purcell factor) and control of emission directivity ${ }^{49,50}$. High-k modes wavevectors values of HMM can reach infinity for semi-infinitely thick HMMs although in reality the numbers are limited by finite thickness of HMM slabs ${ }^{51}$ and inapplicability of effective media approximation to $\mathrm{HMMs}^{52,53}$. Apart from high-k modes supported by HMMs, they offer the following three unique features indispensable for PL enhancement and control:

A. Broadband hyperbolic region.

B. Tunability of hyperbolic region.

C. Directionality by highly anisotropic nature of HMM.

Regarding (A) point above, the hyperbolic region is extremely broad and extends from certain cut-off wavelength and beyond, starting from visible wavelengths to near-infrared ${ }^{54}$ or even to mid-infrared wavelengths ${ }^{55}$, which contrasts with dielectric structures with Mie resonances and plasmonic nanostructures whose resonance is usually confined to certain band of wavelengths. This is the unique feature and clear advant- age of HMMs in order to enhance whole emission bandwidth of emitters. The cut-off wavelength of HMM as is stated in the listed advantage (B) can be tailored for desired wavelength regions by the design of HMMs, such as choice of materials, more specifically the permittivity of metal and dielectric elements and their volume fraction. Regarding the feature $(\mathrm{C})$, the directionality of emission on HMM can also be exploited to create directional emitter in-plane ${ }^{47,48}$ and out of plane of HMM surfaces ${ }^{56}$. These (A) to (C) features are combined to make HMMs an attractive platform for enhancement and control of PL.

Apart from the underlined unique nature of HMMs, we discuss the control of PL in terms of (1) intensity enhancement, (2) lifetime reduction, (3) spectral region, (4) directivity, and (5) lasing. Different configurations of HMMs have been studied for the enhancement of photoluminescence from fluorescence dyes, quantum dots, perovskites and nitrogen-vacancy centers in diamond. In order to describe these features, this review is organized as in the following. We start with introduction of hyperbolic metamaterials and describe their dispersion in wavevector space, which can be categorized as type I and II hyperbolic regions or HMMs. We also overview each type of HMM structures, such as metal-dielectric multilayers, trenches which is the vertical version of metaldielectric multilayers, and nanowires. These are the basic building blocks of HMMs. Out-coupling of enhanced PL emission from metal-dielectric multilayer type HMM is difficult since emitted light is trapped within HMM. Therefore, often, grating out-couplers are integrated into HMM structures and we cover various types of grating out-couplers built on HMMs. As a special case of HMMs, we show PL enhancement by epsilon-near-zero (ENZ) materials. Finally, we discuss the active HMMs with gain media to compensate the absorptive loss of the structures. Throughout the article, we confine ourselves to the light wavelengths ranging from UV to near-infrared, $\lambda=290-1000 \mathrm{~nm}$.

\section{Hyperbolic metamaterials}

In the case of uniaxial anisotropic medium, a relative permittivity tensor $[\varepsilon]$ is expressed by

$$
[\varepsilon]=\left(\begin{array}{ccc}
\varepsilon_{x} & 0 & 0 \\
0 & \varepsilon_{y} & 0 \\
0 & 0 & \varepsilon_{z}
\end{array}\right),
$$

where $\varepsilon_{x}=\varepsilon_{y}=\varepsilon_{\mathrm{o}}$, and $\varepsilon_{z}=\varepsilon_{\mathrm{e}}, \varepsilon_{\mathrm{o}}$ and $\varepsilon_{\mathrm{e}}$ are ordinary and extraordinary permittivities, respectively. When $\varepsilon_{\mathrm{o}}=\varepsilon_{\mathrm{e}}>$ 0 , the medium is an isotropic dielectric, while when 
$\varepsilon_{\mathrm{o}}=\varepsilon_{\mathrm{e}}<0-$ it is an isotropic plasmonic material, for example metal. A HMM or hyperbolic material is a medium when the permittivity components along the principle axes have opposite signs, namely $\varepsilon_{0} \varepsilon_{\mathrm{e}}<0$, and the iso-frequency surface of extraordinary waves given by equation

$$
\left(k_{x}^{2}+k_{y}^{2}\right) / \varepsilon_{\mathrm{o}}+k_{z}^{2} / \varepsilon_{\mathrm{e}}=\omega^{2} / c^{2},
$$

becomes a hyperboloid, where $k_{x}, k_{y}, k_{z}$ are the wavevector components, $\omega$ is the angular frequency and $c$ is the speed of light in vacuum. There are two types of hyperboloid as shown in Fig. 1, which are conventionally classified as: 1) type I HMM: $\varepsilon_{\mathrm{o}}>0$ and $\varepsilon_{\mathrm{e}}<0$, and 2) type II HMM: $\varepsilon_{\mathrm{o}}<0$ and $\varepsilon_{\mathrm{e}}>0$.

In the case of type I HMM, the dispersion band forms a two-fold hyperboloid in the wavevector space (kspace $)^{42}$, as illustrated in Fig 1. Typically, a type I HMM can be realized by a metallic nanowire structure ${ }^{43,57}$, while type II HMM - by a metal-dielectric multilayer stack with deeply subwavelength layers ${ }^{44,58,59}$. Note that in the case of most of naturally occurring materials, both ordinary $\left(\varepsilon_{\mathrm{o}}\right)$ and extraordinary $\left(\varepsilon_{\mathrm{e}}\right)$ permittivity components are of the same sign, resulting in either spherical ( $\varepsilon_{\mathrm{o}}=\varepsilon_{\mathrm{e}}$, isotropic materials) or elliptical iso-frequency surfaces when $\varepsilon_{\mathrm{o}} \neq \varepsilon_{\mathrm{e}}$. However, there are some natural materials that exhibit hyperbolic dispersion for certain wavelength ranges ${ }^{35,36}$.

Materials with hyperbolic iso-frequency contours sup-
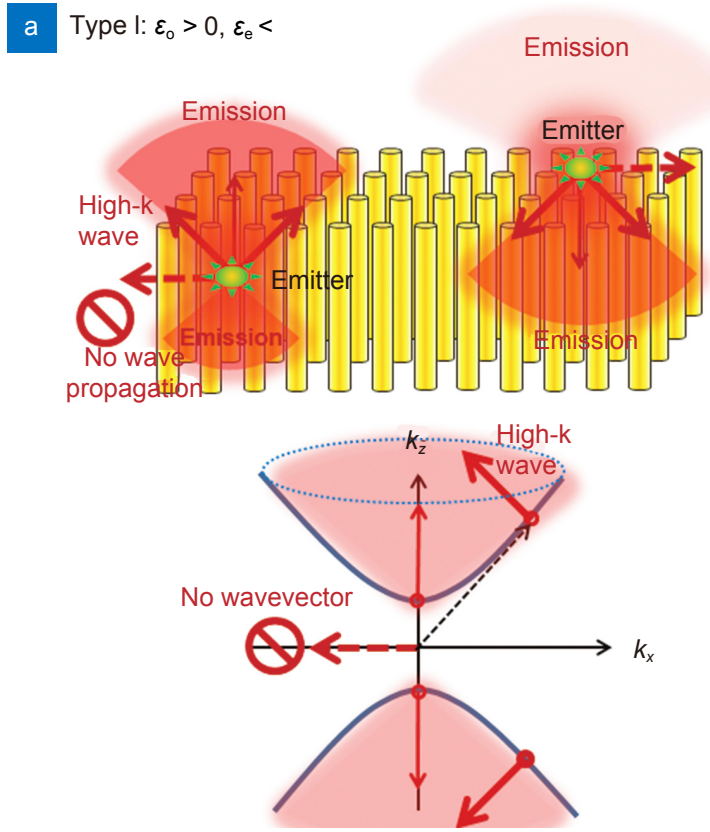

Fig. 1 | Schematic illustration of hyperbolic metamaterials and metasurfaces. (a) Type I hyperbolic metamaterials $\left(\varepsilon_{\mathrm{o}}>0\right.$ and $\left.\varepsilon_{\mathrm{e}}<0\right)$ in metallic nanorod or nanowire configuration and their representative dispersion in the wavevector space (k-space). (b) Type II hyperbolic metamaterials $\left(\varepsilon_{0}<0\right.$ and $\left.\varepsilon_{e}>0\right)$ in metal-dielectric multilayer configuration and their dispersion in the wavevector space. (high-k waves) and correspondingly short effective wavelengths. However, for certain directions in the $\mathrm{k}$ space, there are no any wavevectors available, and, therefore, propagation of light in such direction is prohibited. The high-k lightwaves within HMMs are called bulk plasmon polaritons, BPPs (sometimes also called as volume plasmon-polaritons), as well as hyperbolic surface waves (sometimes referred to as Dyakonov plasmons) that are supported on the top surface of $\mathrm{HMMs}^{47,48,60}$. BPPs propagate and are tightly confined within HMM structures ${ }^{5,57,61}$. Such high-k waves in metal-dielectric multilayer systems are known to originate from the hybridization of short-ranged surface plasmon polaritons supported in each of the dielectric-metaldielectric set ${ }^{61,62}$. Usually, BPPs exhibit highly localized intense electric fields, which together with a non-resonant (broadband) spectral range of their existence, makes them rather convenient tools for the enhancement of PL. A single emitter as a source of PL can be considered as a point dipole that emits light in the form of both propagating (far-field) and evanescent (near-field) waves. In free space, emitted light transfers into far-field propagating waves, and the emitter relaxes by losing energy. However, in the vicinity of a HMM, the near-field components of emitted light are more efficiently coupled to the evanescent (near-field) components of BPPs with

b Type II: $\varepsilon_{\mathrm{o}}<0, \varepsilon_{\mathrm{e}}>0$
$\mathrm{OA}$

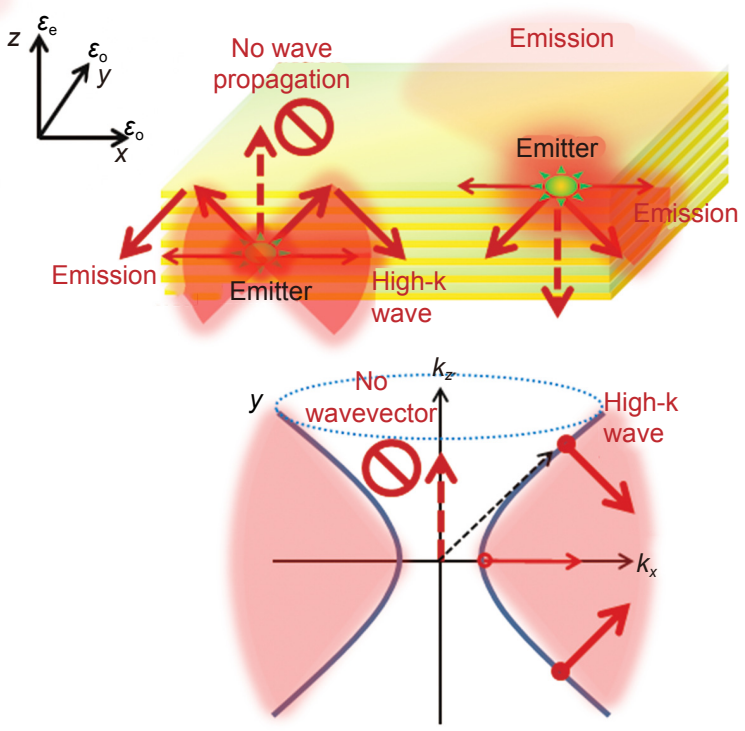


wavenumbers higher than those of the far-field propagating waves, creating a new decay mechanism for the emitter, since the emitted energy is directly sucked by the BPP modes. This results in the reduced lifetime of emitters in comparison with the free space situation. A typically large number of BPP modes supported by HMMs produces an increased photonic density of states (PDOS), leading to a broadband enhancement of the spontaneous emission, referred to as Purcell's effect ${ }^{49,50,63,64}$. In order to characterize the PL enhancement, either the PL intensity or the emission life time (or both) measurement as opposed to the reference emitter samples on a glass substrate or on metal films are typically employed.

\section{Metal-dielectric multilayers}

Metal-dielectric multilayer HMMs have been studied and realized extensively due to the relative ease of fabrication. Their layer thicknesses should be sufficiently smaller than the wavelength of light that they can be characterized by effective parameters, i.e. effective permittivity tensor. By design of a metamaterial (i.e. choosing materials, thicknesses of each layer, filling fraction), the effective dielectric functions or permittivities can be tuned to hyperbolic iso-frequency surface in the wavelength region of interest (see Fig. 1). So, to characterize the optical properties of a HMM, the first step is to find its effective permittivities, and the simplest approach is based on the effective medium approximation (EMA) ${ }^{38,65,66}$. In the case of metal-dielectric multilayers, the effective ordinary and extraordinary permittivities, $\varepsilon_{0}, \varepsilon_{\mathrm{e}}$ are expressed by

$$
\begin{gathered}
\varepsilon_{\mathrm{o}}=f_{\mathrm{m}} \cdot \varepsilon_{\mathrm{m}}+f_{\mathrm{d}} \cdot \varepsilon_{\mathrm{d}}, \\
\varepsilon_{\mathrm{e}}=\left(\varepsilon_{\mathrm{m}} \cdot \varepsilon_{\mathrm{d}}\right) /\left(f_{\mathrm{d}} \cdot \varepsilon_{\mathrm{m}}+f_{\mathrm{m}} \cdot \varepsilon_{\mathrm{d}}\right),
\end{gathered}
$$

where $\varepsilon_{\mathrm{m}}, \varepsilon_{\mathrm{d}}, f_{\mathrm{m}}$, and $f_{\mathrm{d}}$ are the permittivities of the metal and dielectric, and filling factors of metal, and dielectric layers in the multilayer structure, respectively. The light emitters placed into the near-field of optical modes supported by HMMs release their energy predominantly via radiative emission and excitation of plasmonic modes and other non-radiative channels, where usually plasmonic modes dominate as the decay channel.

The first experimental evidence of fluorescence enhancement was demonstrated on a multilayer HMM that consist of 16 stacked layers of gold ( $\mathrm{Au}, 19 \mathrm{~nm})$ and alumina $\left(\mathrm{Al}_{2} \mathrm{O}_{3}, 19 \mathrm{~nm}\right)$ with a 21 -nm thick epoxy with dispersed rhodamine $800(\mathrm{Rh} 800)^{67}$, where 1.8 -fold reduction of the lifetime was reported. The control of spontan- eous emission with multilayer HMMs with alternating metal and dielectric layers was described in ref. ${ }^{68}$. Several different samples were fabricated for the sake of comparison, such as $\operatorname{Ag}(25 \mathrm{~nm}, 11$ layers $) / \operatorname{PMMA}(30 \mathrm{~nm}, 10$ layers), $\mathrm{Ag}(30 \mathrm{~nm}, 5$ layers $) / \mathrm{LiF}(40 \mathrm{~nm}, 4$ layers $), \operatorname{Ag}(30$ $\mathrm{nm}) / \mathrm{LiF}(40 \mathrm{~nm})$ with 8 periods, $\mathrm{Ag}(20 \mathrm{~nm}) / \mathrm{MgF}_{2}(30$ $\mathrm{nm})$ with 8 periods. Two types of dyes were exploited for PL study: IR-140 dye, whose emission peak lies at $\lambda \approx 850$ $\mathrm{nm}$, as well as rhodamine G6 (R6G). Compared with the reference glass substrate, shortening of the luminescence life-times was observed for all samples. For the $\mathrm{Ag} / \mathrm{MgF}_{2}$, the best result was 1.4-times shortening, for $\mathrm{Ag} / \mathrm{LiF}$ - 1.5times, and for Ag/PMMA - 5.7-times.

Apart from Ag layers, Au has been extensively used as the constituent of multilayer HMM structures. HMMs that consist of 16 stacked layers of gold $(\mathrm{Au})$ and alumina $\left(\mathrm{Al}_{2} \mathrm{O}_{3}\right)$ on a glass substrate with a 21-nm dye thin film of epoxy mixed with rhodamine 800 (Rh800) at a $100 \mu \mathrm{M}$ concentration spin coated on top of the spacer layer were realized for fluorescence enhancement ${ }^{69}$. For comparison purposes, two control samples with single thick $(300 \mathrm{~nm})$ and thin $(20 \mathrm{~nm})$ gold layers were also fabricated. In addition, a reference dye thin film was prepared on a bare glass substrate. The dye concentration in the thin film affects the dye parameters. At high concentrations, above $100 \mathrm{M}$, the fluorescence lifetime in dyeepoxy solution is rapidly reduced due to quenching processes, while at low concentrations the fluorescence lifetime is independent of concentration. In thin films of rhodamine 800 embedded in an epoxy matrix, the quantum yield of the dye gradually increases with decreasing concentration. In the experiments, a $100 \mu \mathrm{M}$ concentration was chosen to avoid concentration quenching and to obtain better performance with a higher quantum yield. The PL signal for the $89-\mathrm{nm}$ spacer layer is about 9.3 times stronger for both the HMM and the thick gold substrates (compared with the bare glass substrate), while the thin gold film provides about 6.4 times enhancement.

Enhancement of photoluminescence from colloidal core-shell QDs of cadmium selenide and zinc sulfide (CdSe/ZnS) in proximity with a multilayer HMM was demonstrated ${ }^{54}$. Quantum dots were placed on top of the structure, consisting of alternating layers of silver ( $\mathrm{Ag}, 9$ $\mathrm{nm}$ thick) and titanium dioxide $\left(\mathrm{TiO}_{2}, 22 \mathrm{~nm}\right)$ as shown in Fig. 2. Time-resolved PL measurements were carried out on the metamaterial sample, the control sample, and the glass substrate (Fig. 2(c, d)) at the anticipated 

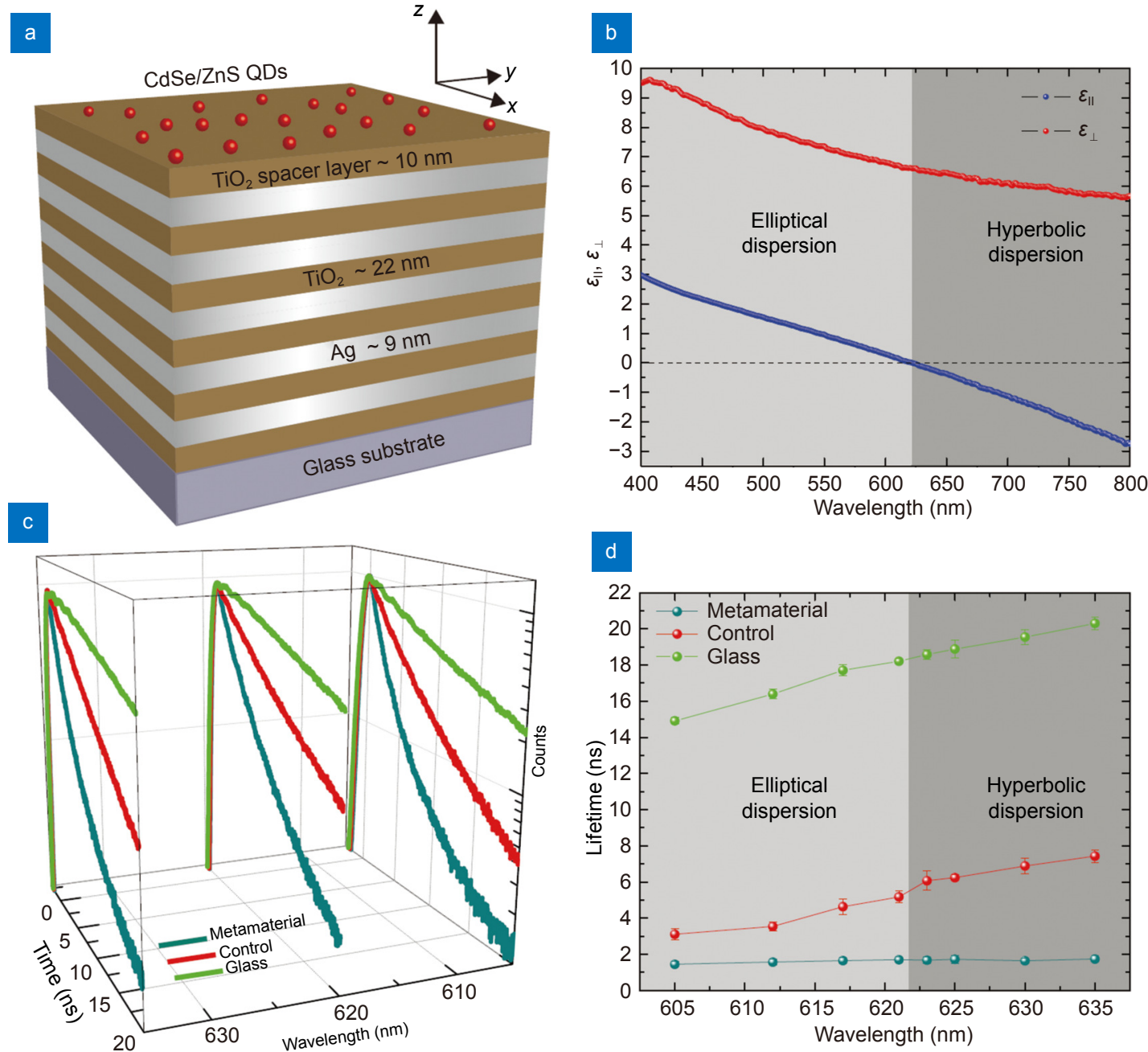

Fig. 2 | Photoluminescence enhancement by metal-dielectric multilayer HMMs. (a) Schematic illustration of Ag-TiO ${ }_{2}$ multilayer $\mathrm{HMM}$ structures. (b) The real part of effective ordinary and extraordinary permittivities. (c) Time-resolved photoluminescence from QDs deposited on the HMM, control sample, and glass substrate at 605,621 , and $635 \mathrm{~nm}$. (d) Lifetime of the QDs as a function of wavelength on the HMM, control sample, and glass substrate. Figure reproduced with permission from ref. ${ }^{54}$. American Association for the Advancement of Science (AAAS).

transition wavelength (elliptic-to-hyperbolic regime, at $621 \mathrm{~nm}$ ) and on both sides from it (605 and $635 \mathrm{~nm}$ ). When compared with the control sample, the metamaterial enhanced the spontaneous emission rate by a factor of 3 at the transition wavelength and by a factor of 4.3 deeper in the hyperbolic regime $(635 \mathrm{~nm})$. The overall reduction in the lifetime of the QDs when compared with those on a glass substrate is $11 \mathrm{~ns}$. The lifetime of the QDs increases as a function of wavelength on both the glass substrate and the control sample [Fig. 2(d)]. This is due to the size distribution of QDs and the dependence of the oscillator strength on energy. The metamaterial sample exhibits a decrease in the lifetime as a function of wavelength in the hyperbolic regime, while such variation is very small in the elliptical spectral region. The lifetimes of QDs on both structures with 1 and
10 bilayers are almost the same in the elliptical spectral region, but significantly differ above the critical wavelength. The large change in the spontaneous emission lifetime of the QDs on the metamaterial compared with the glass substrate and 1 bilayer case is explained by excitation of the high-k BPPs in the metamaterial, as well as by the nonradiative contribution of the surface plasmon polariton (SPP) modes at the metamaterial interface.

Directional PL enhancement of dyes on multilayer HMMs was studied for the sample consisting of 20 alternating layers (10 periods) of $\mathrm{Au}$ (15 $\mathrm{nm}$ thick) and $\mathrm{Al}_{2} \mathrm{O}_{3}$ (28 nm thick) ${ }^{70}$. A dye-dissolved PMMA layer was deposited on top of the $\mathrm{HMM}$, separated by an $\mathrm{Al}_{2} \mathrm{O}_{3}$ spacer layer of thickness $12 \mathrm{~nm}$ from the HMM interface. Coumarin 500 was selected as the organic dye that emit 
light at wavelength $480 \mathrm{~nm}$. The structure showed a 2fold enhancement of spontaneous emission during timeresolved photoluminescence measurements. A similar experiment was conducted on $\mathrm{Au}-\mathrm{Al}_{2} \mathrm{O}_{3}$ multilayer HMMs with $19 \mathrm{~nm}$ thick metal and dielectric layers, where 3 times reduction in emission lifetime of nitrogen vacancy centers (NVCs) in nanodiamonds was demonstrated $^{71}$. The enhancement of single -photon emission from NVCs in nanodiamonds was also observed for a planar multilayer metamaterial that consisted of CMOScompatible ceramics: titanium nitride (TiN) and aluminum scandium nitride $\left(\mathrm{Al}_{x} \mathrm{Sc}_{1-x} \mathrm{~N}\right)^{72}$. Such structure improved the emission properties of a single NVC nanodiamond placed on top. Lifetime reduction was from $17 \mathrm{~ns}$ on a reference sample to $4.3 \mathrm{~ns}$ on the HMM on average. The Purcell factor corresponded to the value 4 . Moreover, it was found that the collected emission power for NVCs near the HMM was increased by a factor of 1.8 on average, although a quite remarkable enhancement of 4.7 was detected in one particular case. A TiN-based multilayer HMM with 10 alternating layers (5 periods) of $\mathrm{TiN}$ and $\mathrm{SiO}_{2}$ deposited on a glass substrate was used for the enhancement of QD emission ${ }^{73}$. A $20 \mathrm{~nm}$ thick Si QDs layer was deposited on the top of the HMM and up to 1.6-times enhanced emission decay rate was observed in respect with identical QDs deposited on silica glass. Up to the 4.4-fold fluorescence enhancement from the nanocomposites made of CdTe QDs monolayers placed between layers of gold nanoparticles was demonstrated ${ }^{74}$. Later on, it was theoretically demonstrated that such structures represents itself a hyperbolic metamaterial and thus is able of increasing the radiative decay rate of emission centers placed inside the structures ${ }^{75}$. Depending on the geometric parameters, such as thickness of the spacer layer, the number of quantum dots and nanoparticle layers, effective permittivity tensor of the entire nanocomposite may become indefinite or hyperbolic. This fact leads to the rise in the photonic density of states, in turn resulting in strong enhancement and pronounced polarization anisotropy of QDs luminescence. Enhancement of UV emission from semiconductor nanoparticles ${ }^{76}$ and multiple quantum wells (MQWs) $)^{77,78}$ deposited on HMMs was also demonstrated.

Upper-excited state emission from molecules is not usually observed owing to strong competition with much faster nonradiative relaxation pathways. However, the radiative decay rate can be increased by modifying the PDOS. Zinc tetraphenylporphyrin (ZnTPP) molecules as emitters embedded in a HMM were shown to enable a 18 -fold increase in fluorescence intensity from the second singlet excited state relative to that from the lowest singlet excited state ${ }^{79}$. Varying the number of periods of the HMM stack enables systematical tuning of the ZnTPP fluorescence spectrum from red (dominated by emission from lowest singlet excited state) to blue (dominated by emission from the second singlet excited state).

Spin-coated organic thin films based on the quinoidal oligothiophene derivative (QQT(CN)4) were demonstrated to exhibit hyperbolic dispersion over a wide spectral range from 670 to $920 \mathrm{~nm}^{80}$. To study the influence of QQT(CN)4 dispersion on the Styryl 9M light-emitting dye molecules, blend films containing a polyvinylpyrrolidone (PVP) host doped with dye molecules were deposited on top of three different substrates: (1) fused silica (FS), (2) $80 \mathrm{~nm}$ thick dielectricmetal HMMs containing $4 \mathrm{Ag} / \mathrm{Al}_{2} \mathrm{O}_{3}$ pairs and (3) organic monolithic natural hyperbolic materials based on a $60 \mathrm{~nm}$ thick QQT(CN)4 film. To gain further insights, polyvinyl alcohol (PVA) layers (spin-coated from water) with thicknesses of 25 and $100 \mathrm{~nm}$ were also inserted between the three types of substrates and the PVP blends. The photoluminescence exhibited slower dynamics at longer wavelengths. The fluorescence decays were described by the sum of two exponential functions. The presence of the two fluorescence lifetimes were attributed to the presence of Styryl $9 \mathrm{M}$ monomers and higher aggregates in the PVP blends. The Purcell factor was measured to be around 1.3 and 1.4 on top of the QQT(CN)4 and HMM substrates correspondingly.

It is well-known that the effective medium theory applied for characterization of dielectric multilayer system and HMMs can experience a breakdown, depending on the thickness of each layer, number of periods, etc. ${ }^{53,81}$. Recently the optimum number of periods of a multilayer HMM for the enhancement of PL from a quantum emitter was theoretically analyzed ${ }^{82}$. In practical system, the number of periods is finite and it is essential to design a multilayer HMM with the optimum number of periods to enhance PL by achieving the highest PDOS. In this work, HMMs consisting of 3, 5, and 8 periods of $\mathrm{Au}$ (15 $\mathrm{nm}) / \mathrm{SiO}_{2}(25 \mathrm{~nm})$ pairs with a quantum emitter, placed inside and in near vicinity of the HMM surface were studied. Modeling confirmed that multilayer HMM slabs with lower number of periods, 3 periods in this study, provide higher total PDOS, giving rise to larger transition rate enhancement. The unit cell thickness can also 
affect enhancement of quantum dots emission ${ }^{83}$. The total thickness of Ag/ITO HMMs was kept $320 \mathrm{~nm}$, while the unit cell thickness ranges from 20 to $80 \mathrm{~nm}$. The study suggested that the Purcell factor increases as the unit cell thickness decreases, exhibiting the maximum enhancement factor of about 40. Related analysis was done in work ${ }^{84}$, where the number of layers and their thickness were varied to study changes of the Purcell factor. The HMM was composed of alternate silver and silicon layers with a dipolar emitter on top of it. It was shown that the multilayer structures with a few layers of small thickness outperform those with many layers of large thickness in terms of the Purcell effect. Such effect was attributed to the larger wavevectors and localization of modes due to the stronger coupling between short range SPPs on thinner metal layers.

Unlike conventional planar multilayer HMMs, HMMs with a curved surface demonstrated an efficient outcoupling of nonradiative modes due to the gradual tapering of HMMs thickness down to few $\mathrm{nm}$. Such outcoupling leads to enhanced spontaneous emission ${ }^{85}$. High-k plasmonic modes propagate along the curved surfaces are then outcoupled into radiative modes and emitted into the far field, realizing a directional light emission with the maximal fluorescent intensity. Detailed simulations revealed a high Purcell factor and a spatial power distribution in the curved HMM, which agreed with the experimental result. The HMMs consist of 20 stacked layers (10 periods) of $\mathrm{Ag}$ and $\mathrm{TiO}_{2}$ deposited on top of a tapered glass capillary. On top of the uppermost Ag layer, an extra 10-nm-thick $\mathrm{TiO}_{2}$ spacer was deposited to decrease the dye absorption. The fabricated HMM has a gradient in its thickness due to the directional deposition. Subsequently, the HMM-capped tapered glass capillary was dipped into the solution of R6G with a concentration of $100 \mu \mathrm{M}$. The PL-intensity distribution indicates enhancement of both its directionality and efficiency. Such curved HMMs, maintaining excellent property in the Purcell effect, can achieve an 80-fold maximum intensity enhancement for directional emission compared with planar HMMs. Another flexible HMM that can be folded was realized by $\mathrm{Au}$ and polymer (PVA) layers, with water-soluble characteristics ${ }^{86}$. These transient HMMs devices can be easily washed away, disappearing with just few drops of deionized water at room temperature. Two samples were made with different fill-fractions of Au: $37.31 \%$ (marked as the HMM1) and 26.87\% (marked as the HMM2), and various dye molecules em- bedded inside PMMA. The photoluminescence kinetics was measured to explore the spontaneous emission effect. The best enhancement of 5.55 (3.80) times for the HMM1 (HMM2) was achieved for the R6G dye molecules.

In order to enhance emission to far-field, metallic cylinder patch antennas can be integrated with HMM structures $^{87,88}$. As an example, a 5-layered planar HMM structure consisting of $\mathrm{Au}$ and zinc sulfide $(\mathrm{ZnS})$ layers with a cylindrical Au patch antenna on the top was modeled ${ }^{87}$. Silicon carbide ( $\mathrm{SiC}$ )-based emitters with emission peaks around $900 \mathrm{~nm}$ were placed inside the HMM. For dipole orientation perpendicular to the interface, the HMMcoupled antenna leads to a spontaneous emission enhancement with a Purcell factor on the order of 400 at $850 \mathrm{~nm}$ and 300 at $680 \mathrm{~nm}$. A similar order of enhancement was actually reported throughout the broad spectral range of 650-1000 nm. Similarly, a cylindrical Ag patch antenna on top of HMM structures with 5, 9, 13, and 17 periods of $\mathrm{Ag}(24 \mathrm{~nm})$ and $\mathrm{TiO}_{2}(30 \mathrm{~nm})$ layers was optimized for dipole emission at $660 \mathrm{~nm}^{88}$. After characterization, the best 200 -fold emission enhancement of CdSeS/ZnS core-shell QDs embedded inside the HMM was achieved for the 5-layered structure. This tendency of better enhancement by fewer periods of HMMs confirms conclusions stemmed from the theoretical analysis ${ }^{82}$.

Optical cavities or resonators made of dielectric materials can trap photons and support strong electric fields, however, the smallest size of such cavities is limited to the order of effective wavelengths of light, $\lambda / n$, where $n$ is the refractive index of the dielectric. In case of HMMs with high-k (large mode refractive index) BPPs, the effective wavelength can be small and hence the size of cavities made of HMMs can be deeply subwavelength, much smaller than conventional dielectric cavities. Such confinement leads to smaller mode volumes and, hence, more intense electric fields in the nanocavities, resulting in the enhancement of the emission rate. An HMM nanocavity was initially demonstrated for 2 to 4 periods multilayers HMMs curved into pyramid shape ${ }^{89}$. Recently, HMM resonators were used for the enhancement of emission from a transition metal dichalcogenide (TMDC) monolayers, $\mathrm{WS}_{2}$, in order to boost light-matter interactions with an atomically thin TMDC film as illustrated in Fig. $3^{90}$. The authors demonstrated about 30fold enhancement of the overall photoluminescence emission intensity from a $\mathrm{WS}_{2}$ monolayer that is placed 


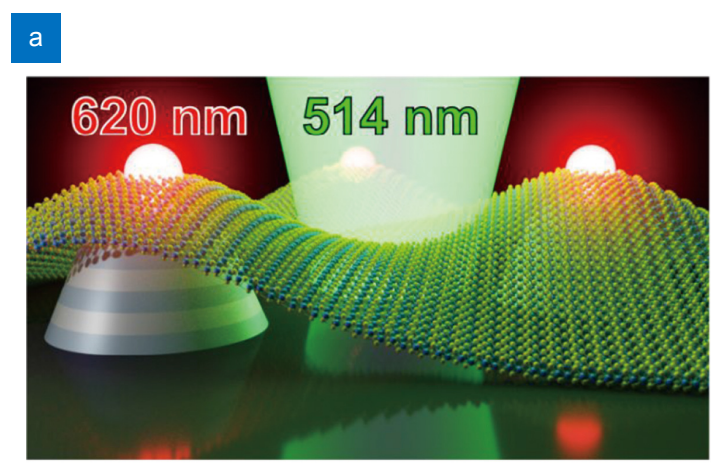

d

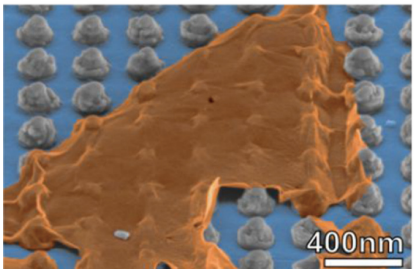

e

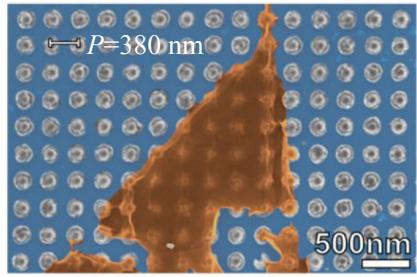

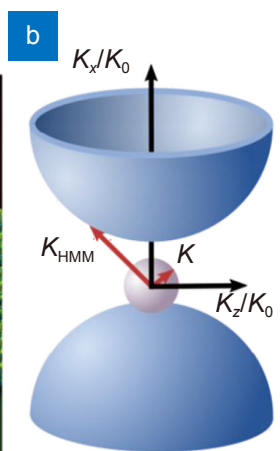

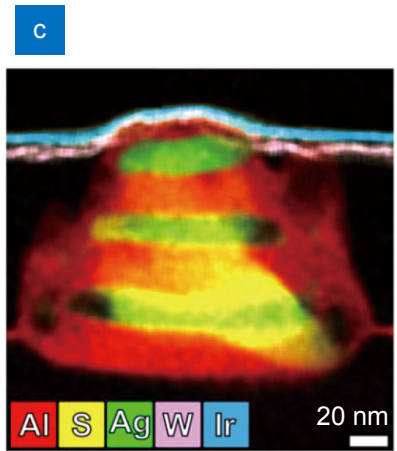

f

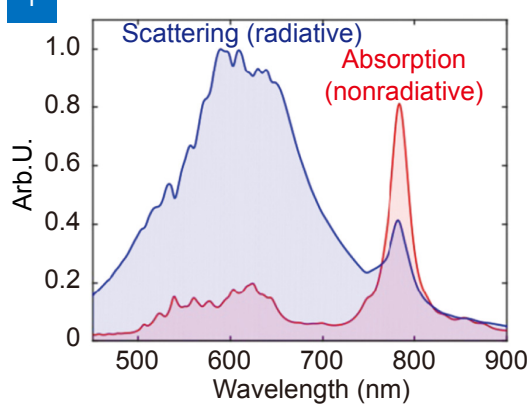

Fig. 3 | Photoluminescence enhancement by metal-dielectric multilayer HMM cavities. (a) Schematic illustration of the WS $S_{2}$ monolayer on the HMM cavities. (b) Type I hyperbolic iso-frequency contour of the HMM. (c) Scanning transmission electron microscopy (STEM) image of the cross section of a $\mathrm{WS}_{2}$ monolayer on a HMM cavity with chemical composition analysis. (d,e) Scanning electron microscopy (SEM) images of HMM cavities with pseudo colors. (f) Normalized calculated scattering and absorption at cross sections of the HMM cavities with a diameter of $160 \mathrm{~nm}$ and a pitch of $380 \mathrm{~nm}$ at the bottom. Figure reproduced with permission from ref..$^{90}$, American Chemical Society.

on top of the HMM resonators. Remarkably, the structure enhances both the excitation (absorption) at $514 \mathrm{~nm}$ and radiative decay rate at $620 \mathrm{~nm}$.

Förster resonance energy transfer (FRET) is the nonradiative transfer of excited state energy from one fluorophore (donor) to another fluorophore (acceptor) via a dipole-dipole coupling process ${ }^{91,92}$. FRET for donor-acceptor pairs located on top of a HMM was experimentally studied for the environments with high local densities of photonic states ${ }^{93}$. Authors observed strong collective interactions of the dye molecules and surface plasmon polaritons, which can affect the absorption and emission spectra and, correspondingly, influence the rate of the Förster energy transfer.

Apart from fluorescence dye, quantum dots, and NVCs, nanocrystals of lead halide perovskite, namely $\mathrm{CsPbI}_{3}$, were used as emitters placed on top of HMMs to study the enhancement of the Purcell factor ${ }^{94}$. The multilayer HMM contained 6 or 8 periods of $\mathrm{Ag}(25 \mathrm{~nm}$ or $40 \mathrm{~nm}$ ) and $\mathrm{LiF}\left(35 \mathrm{~nm}\right.$ or $40 \mathrm{~nm}$ ) with $\mathrm{CsPbI}_{3}$ placed on a $10-50 \mathrm{~nm}$ thick spacer on top of the HMMs. $\mathrm{CsPbI}_{3}$ nanocrystals with size distribution of 11-16 nm emits light at around $520 \mathrm{~nm}$ when excited by $405 \mathrm{~nm}$ laser radiation, 3-fold reduction of emission lifetime was ob- served when the nanocrystals were placed on top of the HMMs. Interestingly, very similar enhancement of radiative rate recombination, by almost 3 times, in quasi-2D perovskite thin films of $\mathrm{BA}_{2} \mathrm{Cs}_{3} \mathrm{MA}_{3} \mathrm{~Pb}_{7} \mathrm{Br}_{2} \mathrm{I}_{20}$ composition deposited on top of gold-alumina HMMs of different thicknesses (from 2 to 7 periods) was reported ${ }^{95}$. The Purcell factor was demonstrated to grow almost linearly with the number of periods in contrary to ref. ${ }^{75,81}$. It means that it is necessary to take into account properties of active material since it has significant influence on spontaneous emission process. The Purcell factor also depends on the pump power. For the pump intensity about $0.2 \mathrm{pJ}$, there are no any difference in the rate enhancement produced by 2- and 7-period HMMs. However, with the increase in pumping to $20 \mathrm{pJ}$, there is a significant difference in the Purcell factor: 1.62 versus 3.1 .

Most of multilayer HMMs are fabricated on a rigid planar substrate which cannot be bent. However, recently multilayer HMMs on flexible rollable paper substrate have been demonstrated ${ }^{96}$. The fabricated structures consisted of four periods of $\mathrm{Au}(25 \mathrm{~nm})$ and PMMA polymer (30 or $40 \mathrm{~nm}$ ) multilayers deposited on a flexible PDMS intermediate layer and rollable paper 
substrate. The structures can be rolled up to the curvature radius of $1 \mathrm{~mm}$ without performance degradation. The emitter is an organic-inorganic perovskite nanocrystal, $\mathrm{CH}_{3} \mathrm{NH}_{3} \mathrm{PBr}_{3}$, which emits light in the visible wavelengths of $520-550 \mathrm{~nm}$. In the paper, 3.5 times enhancement of emission intensity was reported. This new type of HMMs was used to demonstrate enhancement of stimulated emission, as well as laser action.

Emission of quantum dots can be strongly enhanced via coupling to aperiodic metal-dielectric multilayers. Two such cases are reported in literature ${ }^{97,98}$. In both cases, colloidal CdSe/ZnS quantum dots were placed on top of aperiodic sequences of metal and dielectric thin films. The decay rate of quantum emitters was characterized by standard lifetime measurements of fluorescence. For comparison characterization was also conducted with $\mathrm{HMM}$ structures composed of $\mathrm{Ag}$ and $\mathrm{SiO}_{2}$ layers with the same metal filling ratio as in the aperiodic stack and thicknesses of $20 \mathrm{~nm}$ each. The sample with aperiodic structure was based on the use of Fibonacci sequence $(\mathrm{FS})^{97}$. The fluorescence decay curves showed a biexponential behavior. The contribution of the faster decay components was dependent on the density of QDs, and it was minimized by reducing their concentration. First, the lifetime of quantum dots near the uniform silver film and HMM samples was checked giving shorter life time for the latter case. This result indicates that the reduction of lifetime for the HMM is not simply the extinction effect (quenching) due to presence of silver, but regards to the presence of high-k modes. Next, the lifetime on the HMM and FS samples was compared, showing stronger reduction of lifetime by the FS sample. This comparison indicates that the combined contribution of larger PDOS for FS is higher than that of the HMM. The experimental results were consistent with simulations showing a 1.35 fold Purcell factor enhancement on the Fibonacci sequence multilayer than on the HMM and 260-nm-thick silver film (control sample) on one particular wavelength.

Another periodic multilayer arranged in a Tue-Morse (TM) sequence (8 layers $A B B A B A A B$, where $A$ and $B$ represent $\mathrm{Au}$ and $\mathrm{SiO}_{2}$ layers with thicknesses of 20 and $80 \mathrm{~nm}$, respectively) was compared with a periodic HMM (4 periods, 8 layers) with the same filling fraction ${ }^{98}$. The photoluminescence measurements were performed for the QDs on the two multilayer stacks and the glass substrate at the emission wavelength of $580 \mathrm{~nm}$. The most substantial reduction occurred on the wavelength $640 \mathrm{~nm}$ with quantified lifetime reduction in 1.45 and 1.33 times for TM multilayer stacks and periodic HMMs respectively.

The summary of the following chapter is presented in Table 1. From the table, one can see materials used for both metal and dielectric layers, emitters, and enhancement factors. The metallic layers are mostly made of either $\mathrm{Ag}$ or $\mathrm{Au}$ and a wide variety of materials are used for the dielectric layers, such as silica $\left(\mathrm{SiO}_{2}\right)$, alumina $\left(\mathrm{Al}_{2} \mathrm{O}_{3}\right)$, titania $\left(\mathrm{TiO}_{2}\right)$, polymers. The number of periods is in the range of 4 to 10. From the data presented in the table it follows that depending on the structure the fluorescence can be enhance from 1.3 to 200 -fold.

\section{Plasmonic trench structures}

Apart from a conventional configuration of horizontal metal and dielectric layers, a HMM can be realized with vertically standing layers (Fig. 4). Such deep subwavelength plasmonic gratings can be characterized as hyperbolic metasurfaces (HMS) ${ }^{99}$ or so-called trench structures (HMMs) ${ }^{55,100}$. The HMS exhibits in-plane anisotropy, which leads to hyperbolic dispersion and supports both directional surface waves and bulk plasmon modes propagating inside of the trenches. An emitter placed on the surface will show emission in specific directions only ${ }^{47,48}$. Ag-based trenches with semiconductor InGaAsP quantum walls as emitters embedded in the trenches were realized as the HMS as shown in Fig. $4^{56}$. The trench HMS displayed extreme absorption and emission polarization anisotropy. Hyperbolic dispersion was verified by finite difference time domain (FDTD) simulations and $>350 \%$ emission intensity enhancement was experimentally observed relative to the bare semiconductor quantum wells.

Recently, a hyperbolic metasurface made of a metalhalide perovskite gain medium infiltrated between $\mathrm{Au}$ trenches was realized ${ }^{101}$. The Au trenches with pitch of either $80 \mathrm{~nm}$ or $120 \mathrm{~nm}$ function as a type-II HMM in the wavelength range of $740-780 \mathrm{~nm}$ were realized. Strong photoluminescence from $\mathrm{MAPbI}_{3}$ perovskite peaking around $770 \mathrm{~nm}$ was observed. Furthermore, the photoluminescence measurements demonstrated highly anisotropic emission from the HMSs with strong polarization dependence in both the emission and absorption due to the highly anisotropic nature of the trench HMS. Perovskites simultaneously function as PL material and as constituent dielectric, hence, a luminescent hyperbolic metasurface could be utilized as a light source with a tailorable emission polarization. Using another 
Table 1 | Summary of photoluminescence enhancement by multilayer HMM structures. Unless noted, emitters are located on the top surface of HMM structures and the works are experimental.

\begin{tabular}{|c|c|c|c|}
\hline HMM structures and materials & $\begin{array}{l}\text { Emitters (emission peak } \\
\text { wavelength) }\end{array}$ & Enhancement factor & $\begin{array}{l}\text { References } \\
\text { (year) }\end{array}$ \\
\hline $\mathrm{Au}(19 \mathrm{~nm}) / \mathrm{Al}_{2} \mathrm{O}_{3}$ & Rhodamine 800 (715 nm) & 1.8-fold reduction of lifetime & ref. $^{67}(2010)$ \\
\hline $\begin{array}{c}\mathrm{Ag}(25 \mathrm{~nm}, 11 \text { layers }) / \mathrm{PMMA}(30 \mathrm{~nm}, 10 \text { layers }) ; \mathrm{Ag}(30 \\
\mathrm{nm}, 5 \text { layers }) / \mathrm{LiF}(40 \mathrm{~nm}, 4 \text { layers }) ; \mathrm{Ag}(30 \mathrm{~nm}) / \mathrm{LiF}(40 \mathrm{~nm}) \\
8 \text { periods, and } \mathrm{Ag}(20 \mathrm{~nm}) / \mathrm{MgF}_{2}(30 \mathrm{~nm}) 8 \text { periods }\end{array}$ & IR-140 dye $(850 \mathrm{~nm})$ & 1.4 for $\mathrm{Ag} / \mathrm{LiF} ; 5.7$ for $\mathrm{Ag} / \mathrm{PMMA}$ & ref. ${ }^{68}(2011)$ \\
\hline $\mathrm{Au}(19 \mathrm{~nm}) / \mathrm{Al}_{2} \mathrm{O}_{3}(19 \mathrm{~nm}), 8$ periods & Rhodamine $800(720 \mathrm{~nm})$ & 9.3 & ref. $^{69}(2012)$ \\
\hline $\mathrm{Ag}(9 \mathrm{~nm}) / \mathrm{TiO}_{2}(22 \mathrm{~nm}), 10$ periods & CdSe/ZnS colloidal QDs (630 nm) & 3 & ref. $^{54}(2012)$ \\
\hline $\mathrm{Au}(15 \mathrm{~nm}) / \mathrm{Al}_{2} \mathrm{O}_{3}(28 \mathrm{~nm}), 10$ periods & Coumarin $500(480 \mathrm{~nm})$ & 2 & ref. $^{70}(2013)$ \\
\hline $\mathrm{Au}(19 \mathrm{~nm}) / \mathrm{Al}_{2} \mathrm{O}_{3}(19 \mathrm{~nm}), 8$ periods & NVC $(637 \mathrm{~nm})$ & 2.57 & ref. $^{71}(2013)$ \\
\hline $\begin{array}{l}\text { Au nanoparticles }(15 \mathrm{~nm}) / \mathrm{CdTe} \text { QDs }(5.5 \mathrm{~nm}) \text {, separated } \\
\text { by dielectric (PDDA/PPS) spacers with varied thickness } \\
(0-10 \mathrm{~nm}), 2-5 \text { periods }\end{array}$ & CdTe QDs (590 nm) & 4.4 & $\begin{array}{l}\text { ref. }^{74,75}(2011, \\
2014)\end{array}$ \\
\hline $\operatorname{TiN}(8.5 \mathrm{~nm}) / \mathrm{Al}_{0.7} \mathrm{Sc}_{0.3} \mathrm{~N}(6.3 \mathrm{~nm}), 10$ periods & NVC $(600-800 \mathrm{~nm})$ & 4.7 max Purcell factor & ref. $^{72}(2015)$ \\
\hline $\mathrm{TiN}(15 \mathrm{~nm}) / \mathrm{SiO}_{2}(15 \mathrm{~nm}), 5$ periods & $20 \mathrm{~nm}$ thick Si QDs $(720 \mathrm{~nm})$ & 1.6 & ref. $^{73}(2015)$ \\
\hline $\mathrm{Ag}(25 \mathrm{~nm}) 7$ layers $/ \mathrm{MgF}_{2}(35 \mathrm{~nm}) 6$ layers & $\begin{array}{l}\text { HITC dye-doped polymeric film } \\
\qquad(860 \mathrm{~nm})\end{array}$ & 7 & ref. $^{78}(2015)$ \\
\hline $\mathrm{Ag}(10 \mathrm{~nm}) / \mathrm{TiO}_{2}(30 \mathrm{~nm}), 10$ periods & Rhodamine 6G (R6G, 540-600 nm) & 80 -fold intensity enhancement & ref. ${ }^{85}(2018)$ \\
\hline $\mathrm{Au}(26.87-37.31 \%) /$ poly(vinyl alcohol) (PVA), 4 periods & R6G dye $(540-600 \mathrm{~nm})$ & 1.55 for HMM1, 1.18 for HMM2 & ref. ${ }^{86}(2018)$ \\
\hline $\begin{array}{l}\mathrm{Ag}(22 \mathrm{~nm}) / \mathrm{MoO}_{3}(10 \mathrm{~nm}) 6 \text { periods, } \mathrm{HMM} ; \\
\mathrm{Ag}(12 \mathrm{~nm}) / \mathrm{MoO}_{3}(20 \mathrm{~nm}) 6 \text { periods, elliptic }\end{array}$ & $\mathrm{ZnO}$ nanoparticles $(395 \mathrm{~nm})$ & $\begin{array}{l}\text { Lasing threshold } 20 \% \text { less and } 6 \\
\text { times emission intensity }\end{array}$ & ref. $^{76}(2018)$ \\
\hline $\mathrm{Al}(20 \mathrm{~nm}) / \mathrm{MgF}_{2}(20 \mathrm{~nm}), 4$ periods & 15-nm thick AIGaN MQWs $(318 \mathrm{~nm})$ & 160 -fold emission rate & ref. $^{77}(2018)$ \\
\hline $\begin{array}{c}\mathrm{Au}(30 \mathrm{~nm}) / \mathrm{ZnS}(30 \mathrm{~nm}) 5 \text { periods, with cylindrical gold } \\
\text { patch antenna }\end{array}$ & $\mathrm{SiC}(900 \mathrm{~nm})$ & $\begin{array}{l}\text { Purcell factor of } 400 \text { at } 850 \mathrm{~nm} \text {. } \\
\text { (Theory) }\end{array}$ & ref. $^{87}(2018)$ \\
\hline $\mathrm{Ag}(25 \mathrm{~nm}) / \mathrm{PMN}$ & $\begin{array}{l}\text { Zinc tetraphenylporphyrin (ZnTPP), } \\
\mathrm{S}_{1}(580-670 \mathrm{~nm}), \mathrm{S}_{2}(400-460 \mathrm{~nm})\end{array}$ & $\begin{array}{l}\text { 18-fold increase in fluorescence } \\
\text { intensity from } S_{2} \text { state to } S_{1} \text {. }\end{array}$ & ref. $^{79}(2018)$ \\
\hline $\begin{array}{l}320 \mathrm{~nm} \text { thick HMM of } \mathrm{Ag} / \mathrm{lTO} \text { with unit cell thickness from } \\
\qquad 20 \text { to } 80 \mathrm{~nm}\end{array}$ & CdSe/ZnS QDs (550 nm) & 40-fold intensity enhancement & ref. $^{83}(2018)$ \\
\hline $\begin{array}{l}\text { Quinoidal oligothiophene derivative } \\
\text { QQT(CN)4 (60 nm thick), } 670 \text { to } 920 \mathrm{~nm}\end{array}$ & Styryl9M dye $(680-850 \mathrm{~nm})$ & 1.3-1.4 Purcell factor & ref. ${ }^{00}(2019)$ \\
\hline $\begin{array}{c}\mathrm{Ag}(24 \mathrm{~nm}) / \mathrm{TiO}_{2}(30 \mathrm{~nm}) 5,9,13 \text {, and } 17 \text { layers with } \\
\text { cylindrical Ag antenna }\end{array}$ & CdSeS/ZnS QDs $(660 \mathrm{~nm})$ & $\begin{array}{l}\text { 200-fold enhancement for 5-layered } \\
\text { HMM }\end{array}$ & ref. ${ }^{88}(2020)$ \\
\hline $\mathrm{Au}(15 \mathrm{~nm}) / \mathrm{SiO}_{2}(25 \mathrm{~nm}) 3,5$, and 8 periods & Emitter $(600-1600 \mathrm{~nm})$ & 60-85 (Theory) & ref. ${ }^{82}(2020)$ \\
\hline $\mathrm{Ag}(16 \mathrm{~nm}) / \mathrm{Al}_{2} \mathrm{O}_{3}(24 \mathrm{~nm}), 3$ periods & $\mathrm{WS}_{2}$ monolayer $(615 \mathrm{~nm})$ & $\begin{array}{l}\text { 30-fold enhancement of the overall } \\
\text { PL intensity }\end{array}$ & ref. ${ }^{90}(2020)$ \\
\hline $\begin{array}{c}\mathrm{Ag}(25 \mathrm{~nm}) / \mathrm{LiF}(35 \mathrm{~nm}) \text { and } \mathrm{Ag}(40 \mathrm{~nm}) / \mathrm{LiF}(40 \mathrm{~nm}), 6 \text { or } 8 \\
\text { periods }\end{array}$ & $\begin{array}{l}\mathrm{CsPb}_{3} \text { Perovskite nanocrystals } \\
\qquad(520 \mathrm{~nm})\end{array}$ & 3-fold Purcell enhancement & ref. ${ }^{44}(2020)$ \\
\hline $\mathrm{Au}(10 \mathrm{~nm}) / \mathrm{Al}_{2} \mathrm{O}_{3}(10 \mathrm{~nm}), 2-7$ periods & $\begin{array}{c}\mathrm{BA}_{2} \mathrm{Cs}_{3} \mathrm{MA}_{3} \mathrm{~Pb}_{7} \mathrm{Br}_{2} \mathrm{I}_{20} \text { Perovskite } \\
\text { film }(700 \mathrm{~nm})\end{array}$ & $\begin{array}{l}\text { 1.6-3-fold Purcell enhancement } \\
\text { depending on number of periods }\end{array}$ & ref. ${ }^{95}(2021)$ \\
\hline $\begin{array}{c}\mathrm{Ag}(25 \mathrm{~nm}) / \mathrm{PMMA}(40 \mathrm{~nm}) \text { and } \mathrm{Ag}(25 \mathrm{~nm}) / \mathrm{PMMA}(30 \mathrm{~nm}), \\
4 \text { periods on paper }\end{array}$ & $\begin{array}{l}\mathrm{MAPbBr}_{3} \text { perovskite nanocrystals } \\
\qquad(520-550 \mathrm{~nm})\end{array}$ & 3.5-fold intensity enhancement & ref. ${ }^{96}(2021)$ \\
\hline $\begin{array}{c}\text { Aperiodic } \mathrm{Ag}(20 \mathrm{~nm}, 6 \text { layers }) / \mathrm{SiO}_{2}(20 \mathrm{~nm}, 6 \text { layers }) \text { in } \\
\text { Fibonacci sequence }\end{array}$ & Colloidal CdSe/ZnS QDs (640 nm) & $\begin{array}{c}1.6 \text { than } \mathrm{Ag} \text { layer, } 1.35 \text { than } \\
\text { periodic material }\end{array}$ & ref. ${ }^{97}(2014)$ \\
\hline $\begin{array}{c}\text { Aperiodic } \mathrm{Ag}(20 \mathrm{~nm}, 8 \text { layers }) / \mathrm{SiO}_{2}(80 \mathrm{~nm}, 8 \text { layers }) \text { in } \\
\text { Tue-Morse (TM) sequence }\end{array}$ & Colloidal CdSe/ZnS QDs (640 nm) & 1.45 than glass substrate & ref. ${ }^{98}(2019)$ \\
\hline
\end{tabular}

perovskite material instead of $\mathrm{MAPbI}_{3}$ could allow for tuning of emission wavelengths.

\section{Nanorod and nanowire structures}

In the case of HMMs consisting of metal nanorods or nanowires with diameters and periods sufficiently smal- ler than that of operating wavelengths, the effective ordinary and extraordinary permittivities of the metamaterial are expressed by

$$
\begin{aligned}
\varepsilon_{\mathrm{o}}=[ & \left.\left(1+f_{\mathrm{m}}\right) \varepsilon_{\mathrm{m}} \cdot \varepsilon_{\mathrm{d}}+\left(1-f_{\mathrm{m}}\right) \varepsilon_{\mathrm{d}}\right] /\left(1+f_{\mathrm{m}}\right) \varepsilon_{\mathrm{d}} \\
& +\left(1-f_{\mathrm{m}}\right) \varepsilon_{\mathrm{m}},
\end{aligned}
$$




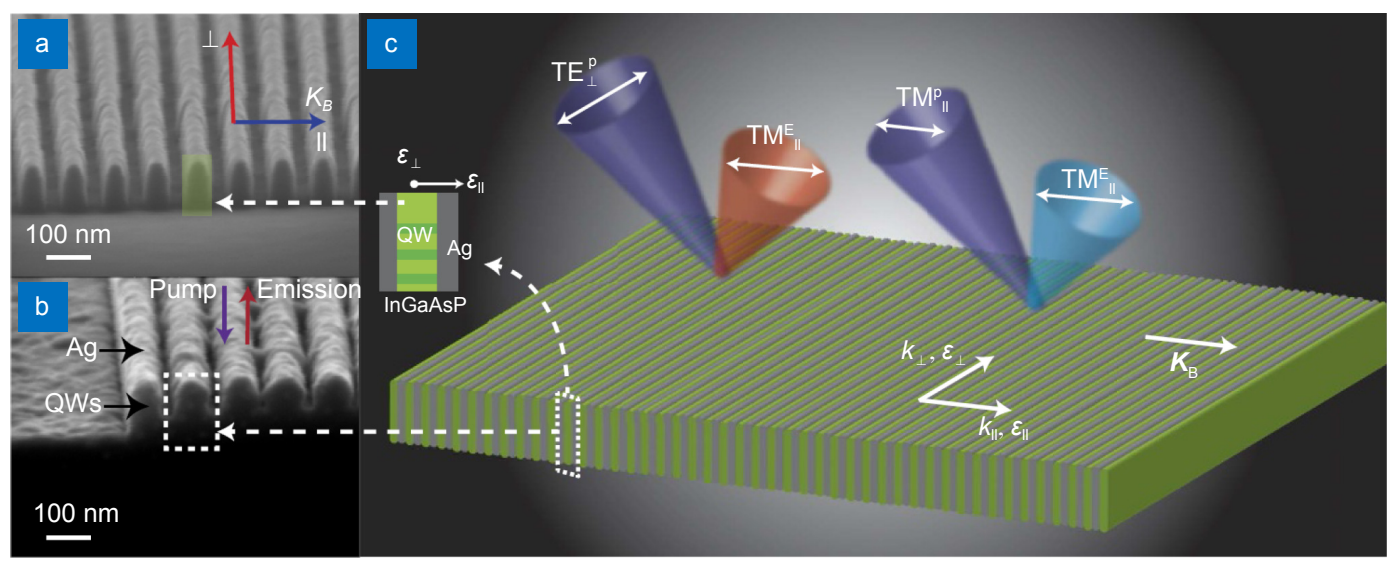

Fig. 4 | Photoluminescence enhancement on trench hyperbolic metasurface. (a,b) SEM images of InGaAsP MQW trenches of $100 \mathrm{~nm}$ height and $40 \mathrm{~nm}$ width, separated by $40 \mathrm{~nm}$ trenches. Ag is deposited by sputtering, partially filling the trenches to create a HMS with $80 \mathrm{~nm}$ period. (c) Illustration of optical pumping with different polarizations $\left(\mathrm{TM}^{\mathrm{P}} \|\right.$ or $\mathrm{TE}_{\perp} \perp$ of the $\mathrm{HMS}$ results in collected emission polarized predominantly parallel $\left(\mathrm{TM}^{\mathrm{E}} \|\right)$ to the metasurface. $\boldsymbol{K}_{\mathrm{B}}$ is the Bloch wavevector. Figure reproduced from ref. ${ }^{56}$, under a Creative Commons Attribution 4.0 International License.

$$
\varepsilon_{\mathrm{e}}=f_{\mathrm{m}} \cdot \varepsilon_{\mathrm{m}}+f_{\mathrm{d}} \cdot \varepsilon_{\mathrm{d}},
$$

where $\varepsilon_{\mathrm{m}}, \varepsilon_{\mathrm{d}}$ and $f_{\mathrm{m}}, f_{\mathrm{d}}$ are the permittivities and volume fractions of metal nanowires and dielectric matrix, respectively.

One of the earliest works on PL enhancement by a HMM was conducted with an array of Ag nanowires in $\mathrm{Al}_{2} \mathrm{O}_{3}$ matrix ${ }^{102}$, where Ag nanowires with $35 \mathrm{~nm}$ diameter occupied $15 \%$ of volume fraction. The emitter was IR-140 laser dye with the maximum radiation at $892 \mathrm{~nm}$. Dye was dispersed in an $80 \mathrm{~nm}$ thick PMMA film deposited on the nanowire HMM structure. A 6-fold reduction of the emission lifetime of the dye was reported. Later, an array of gold nanorods or nanowires with type I hyperbolic dispersion for bulk plasmon modes that propagate inside the metamaterial was used for enhancement of fluorescence, as shown in Fig. $5^{103}$. The structure consisted of $\mathrm{Au}$ nanorods with dimensions: approximately $38 \mathrm{~nm}$ diameter, $150 \mathrm{~nm}$ height, and approximately $80 \mathrm{~nm}$ pitch. Almost 50 -fold reduction of the fluorescence lifetime for the emitters placed inside the HMM was observed in comparison with a 2-3-fold reduction for emitters placed on top of the metamaterial. This work demonstrated that the BPP modes play a significant role in determining the spontaneous emission properties within plasmonic nanorod HMMs. The spectrum and lifetime of the emission can be controlled separately for TM and TE polarizations by coupling to different BPPs supported in the metamaterial slab. Similar spontaneous radiation of an emitter inside nanorod-based metamaterials was studied by the same group ${ }^{104}$. It was not possible to accomplish direct measurement of the specific life- time reduction. A broadband macroscopically averaged lifetime measurements were conducted instead. The averaged lifetime reduction over the sample was of the order of 30 , showing that the real numbers are orders of magnitude higher. Furthermore, the Au nanorod array is employed to increase the FRET rate for donor-acceptor pairs separated by fixed distances $(3.4,6.8$, and $10.2 \mathrm{~nm})$ embedded inside the HMM structure ${ }^{105}$. It was shown that donor-acceptor pairs placed inside the gold nanorod-based metamaterial exhibited a 12-fold increase of the PDOS and a 13-fold increase of the FRET rate compared to those located on bare glass.

Another approach to fabricate nanorod based HMMs was realized with using ion-etching of electrochemically grown Au nanorods, which provides closely packed structures with well-defined and smooth nanocones (cone base 40-60 nm, cone apex $2 \mathrm{~nm}$, nanocone density $\left.10^{10} \mathrm{~cm}^{-2}\right)^{106}$. For the nanorod and nanopencil (nanorod with tapered end) metamaterials, the strongest field enhancement occurs at the wavelength of $596 \mathrm{~nm}$ with the corresponding intensity enhanced by a factor of approximately 40 and 60 , respectively. For the nanocone metamaterials, this occurs at $660 \mathrm{~nm}$ with a 105-fold intensity enhancement. For both nanopencil and nanocone metamaterials, the maximum field enhancement is observed at the apex.

In the specific case of phosphorescence, which is a second-order quantum process, emitters typically exhibit lifetimes in the range of milliseconds to seconds, orders of magnitude longer than the common nanosecond lifetimes of fluorescent dyes. This comes from the 

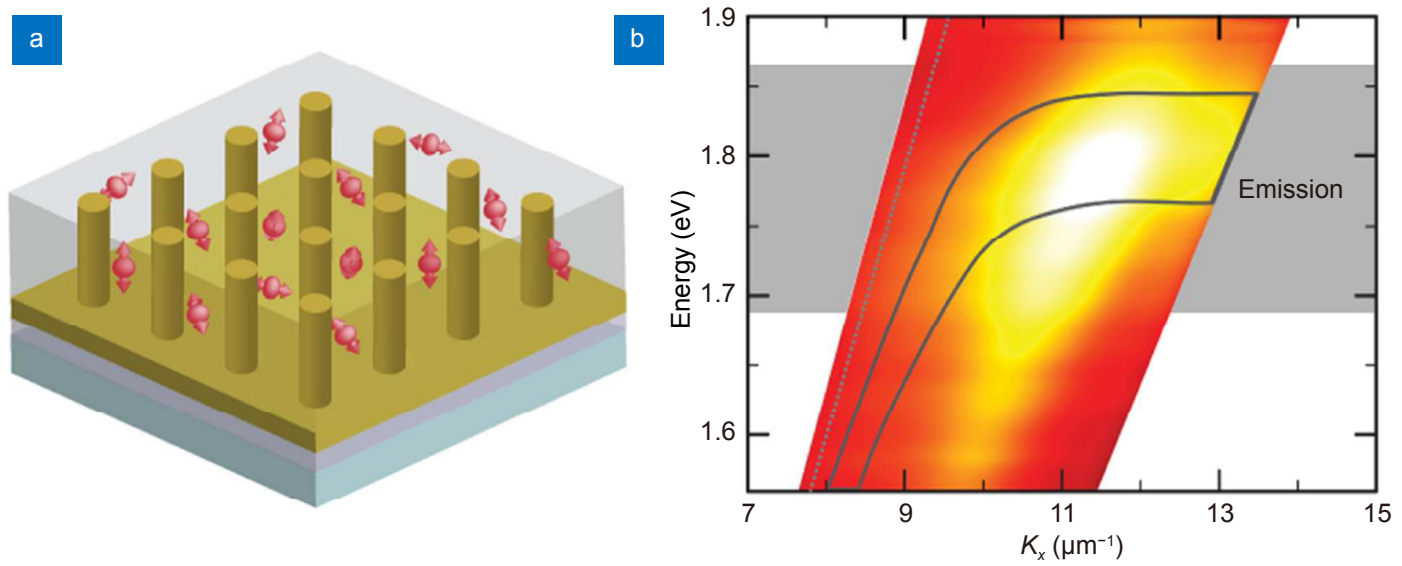

Fig. 5 | Plasmonic nanowire-based HMMs. (a) An array of Au nanorods with approximately $38 \mathrm{~nm}$ diameter, $150 \mathrm{~nm}$ height and $80 \mathrm{~nm}$ spacing embedded in a dye-doped PMMA matrix. (b) Emission in waveguided modes. Experimental dispersions of the photoluminescence $(\mathrm{PL})$ enhancement measured for TM-polarized emission whose position reproduced from the reflection dispersion is shown as shaded area. Gray dotted line is the light line in air, bulk greyed region is the emission band of LD700 dye. Figure reproduced with permission from: ref. ${ }^{103}$, American Chemical Society.

forbidden nature of the transitions involved in the emission process, including transitions between states of different spin multiplicities such as singlet-triplet transitions. The decay rate enhancement of a singlet-triplet transition was investigated for a long lifetime phosphorescent ruthenium-based complex $(\mathrm{Ru}(\mathrm{dpp}))$ inside a gold-nanorod-based hyperbolic metamaterial ${ }^{107}$. Typically, the decay rate enhancement in fluorescence processes follows theoretical predictions by the PDOS theory. However, in the case of singlet-triplet transitions the strong local gradients of the electro-magnetic field in the plasmonic nanostructures need to be taken into account. For example, a 2750-fold decay rate enhancement demonstrated in this publication cannot be explained only by the standard PDOS approach.

The summary of plasmonic nanowire-based HMM structures is given in Table 2. It has been demonstrated that these structures can be used to enhance photoluminescence by more than 30 times. Nanowires are made of either gold or silver with diameters of 38 to $60 \mathrm{~nm}$, periods of 100 to $250 \mathrm{~nm}$, and heights of $150 \mathrm{~nm}$ to 250 $\mathrm{nm}$. The enhancement factors range from 40 to 105 . In addition, nanorods HMMs improve the FRET effectiveness and allow forbidden transitions such as a singlet-triplet transitions in a long lifetime phosphorescent ruthenium-based complex.

Table 2 | Summary of photoluminescence enhancement by plasmonic nanowire HMM structures. Unless noted, emitters are located on the top surface of HMM structures and the works are experimental.

\begin{tabular}{|c|c|c|c|}
\hline HMM structures and materials & $\begin{array}{l}\text { Emitters (emission peak } \\
\quad \text { wavelength) }\end{array}$ & Enhancement factor & $\begin{array}{l}\text { References } \\
\text { (year) }\end{array}$ \\
\hline $\begin{array}{c}\text { Ag nanowires, } 35 \mathrm{~nm} \text { diameter, } 15 \% \text { volume fraction in } \mathrm{Al}_{2} \mathrm{O}_{3} \\
\text { host }\end{array}$ & IR-140 laser dye $(892 \mathrm{~nm})$ & 6 -fold reduction of lifetime & ref. $^{102}(2010)$ \\
\hline Au nanorods, $38 \mathrm{~nm}$ diameter, $150 \mathrm{~nm}$ height, and $80 \mathrm{~nm}$ pitch. & LD700 dye $(700 \mathrm{~nm})$ & 50 & ref. ${ }^{103}(2017)$ \\
\hline $\begin{array}{c}\text { Au nanorods, } 40 \text { and } 25 \mathrm{~nm} \text { diameter, } 250 \mathrm{~nm} \text { height, and } \\
\text { surface densities of } 35 \% \text { and } 14 \%\end{array}$ & $\begin{array}{l}\text { PVA embedded with R101 dye } \\
\qquad(606 \mathrm{~nm})\end{array}$ & 4.6 & ref. $^{108}(2017)$ \\
\hline Silver nanowire-alumina HMM (filling fractions $f=0.15$ and 0.2 ) & $\begin{array}{l}\text { CdSe QDs of diameters } 5 \mathrm{~nm} \text { and } \\
6.5 \mathrm{~nm}(580 \text { and } 670 \mathrm{~nm})\end{array}$ & $\begin{aligned} 2-3 \text { for the } f & =0.15 \text { against } \\
f & =0.2\end{aligned}$ & ref. ${ }^{109}(2017)$ \\
\hline Au nanorod, $50 \mathrm{~nm}$ diameter, $100 \mathrm{~nm}$ pitch, and $250 \mathrm{~nm}$ height & $\begin{array}{l}\text { D1 (fluorescein, } 514 \mathrm{~nm} \text { ), D2 (Alexa } \\
514,550 \mathrm{~nm} \text { ), D3 (ATTO 550, } 575 \\
\mathrm{~nm} \text { ) and D4 (ATTO 647N, } 670 \mathrm{~nm} \text { ) }\end{array}$ & 30 & ref. $^{104}(2017)$ \\
\hline $\begin{array}{l}\text { Au nanorod, } 50 \mathrm{~nm} \text { diameter, } 260 \mathrm{~nm} \text { height, } 100 \mathrm{~nm} \text { inter-rod } \\
\text { spacing }\end{array}$ & $\begin{array}{l}\text { ATTO } 550 \text { and ATTO } 647 \mathrm{~N} \text { dyes } \\
\qquad(554 \mathrm{~nm})\end{array}$ & 13 FRET rate & ref. $^{105}(2018)$ \\
\hline $\begin{array}{l}\text { Au nanorod, } 50 \mathrm{~nm} \text { diameter, } 250 \mathrm{~nm} \text { height, } 100 \mathrm{~nm} \text { inter-rod } \\
\text { spacing }\end{array}$ & $\begin{array}{l}\text { Ruthnium-based phosphorescent } \\
\text { complex (Ru(dpp), } 620 \mathrm{~nm} \text { ) }\end{array}$ & 2750 (Theory) & ref. $^{107}(2019)$ \\
\hline $\begin{array}{c}\text { Au nanorod ( } 60 \mathrm{~nm} \text { diameter and } 110,160 \text {, and } 240 \mathrm{~nm} \text { lengths), } \\
\text { nanocone (cone base } \sim 40-60 \mathrm{~nm} \text {, cone apex }<2 \mathrm{~nm} \text { ), } \\
\text { nanopenscil ( } 60 \mathrm{~nm} \text { base diameter and } 10 \mathrm{~nm} \text { at the top) }\end{array}$ & None & $\begin{array}{c}40,60 \text { at } 596 \mathrm{~nm} \text {, and } 105 \text { at } \\
660 \mathrm{~nm} \text { of the field } \\
\text { enhancement }\end{array}$ & ref. $^{106}(2019)$ \\
\hline
\end{tabular}




\section{Grating out-coupler on HMM}

There are various works on combining a grating on top or inside multilayer and nanowire HMMs. The role of the grating is to out-couple photoluminescence enhanced inside of HMM to far-field since there is a pronounced momentum mismatch between the HMM modes and lightwaves in ambient space, i.e. air superstrate and substrate. A line (1D) grating with pitch $P$ provides the parallel wave vector of

$$
N_{\mathrm{g}}=n \sin \varphi-m \lambda / P(m=1,2,3 \cdots),
$$

where $n$ is the refractive index of the ambient medium, usually air $(n=1)$ and $\varphi$ is the incident angle. When $N_{g}$ equals the effective mode index of BPP modes, emission is straightforwardly out-coupled from the HMMs. The gratings couplers studied here are 1D line grating, 2D gratings made of square lattice or hexagonal lattice of air hole arrays, and circular grating, also known as "bullseye" gratings.

\section{D (line) grating out-coupler}

The spontaneous emission rate of a dye molecule embedded in a polymeric matrix can be greatly enhanced by a nanostructured HMM composed of alternating multilayers of $\mathrm{Ag}$ and $\mathrm{Si}^{110}$. To demonstrate this, four samples (Fig. 6) were used: bulk silver slab, $\mathrm{Ag}$ grating, $\mathrm{Ag} / \mathrm{Si}$ $\mathrm{HMM}$, and Ag/Si HMM with grating. By adjusting the filling ratio of $\mathrm{Ag}$ in HMMs, the Purcell enhancement can be tuned to achieve better control of emission processes at desired wavelengths, demonstrating the versatility of plasmonic PDOS engineering in multilayer HMMs. The lifetime of R6G dye molecules on various samples was characterized using resolved luminescence time. As a reference, the lifetime in a methanol solution was measured to be $3.8 \mathrm{~ns}$ with a mono-exponential fit. For the $\mathrm{Ag} / \mathrm{Si} \mathrm{HMM}$ with $200 \mathrm{~nm}$ period grating, the lifetime is reduced by almost one order of magnitude compared to the bare silver grating. In total, there is an increase in decay rate by 54 times compared with the dye on a $\mathrm{Si}$

a

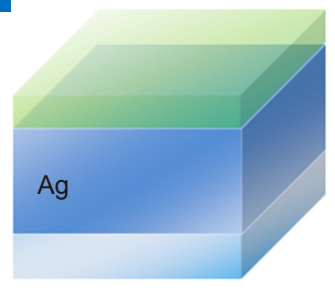

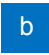

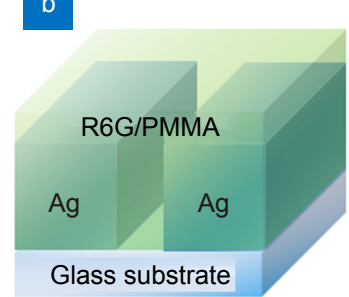

film. The increase in the decay rate is observed in the case of $\mathrm{Ag} / \mathrm{Si} \mathrm{HMM}$ with grating compared with the signal from the dye in PMMA on the surface of a bulk silver slab or Ag/Si HMM. The decay rate and luminescence intensity are growing with shortening the lattice period. The lifetime is minimal for the period of $80 \mathrm{~nm}$ (in 76 times). A luminescence enhancement coefficient close to 80-fold was achieved for HMM with a period of $80 \mathrm{~nm}$. Shorter lattice periods are better suited to excite high-k bulk plasmon modes, what leads to a simultaneous increase in both the Purcell factor and fluorescence intensity. For the 1D grating with the period of $200 \mathrm{~nm}$, the averaged 120 -fold intensity enhancement and local enhancement up to 1000-fold at some positions was observed. Later, in a theoretical work on the systematic optimization conducted by the same group ${ }^{11}$, they studied the multilayer HMMs that consisted of 10 layers of Ag and 11 layers of $\mathrm{Si}$ with a period of $20 \mathrm{~nm}$ on a glass substrate to find optimal material combinations for Purcell enhancement. Large emission intensity with up to 120 fold enhancement was shown by comparing different geometry parameters, the emitter distance and their wavelengths.

To demonstrate the Purcell effect of a pristine and patterned HMMs, Fig. 7(a, b), time-resolved photoluminescence decay measurements of QDs on a Ag-SiO ${ }_{2}$ multilayer stack and glass substrate (control case) were conducted. Characterization of CdSe/ZnS QDs spontaneous emission rate was performed in a broad wavelength range from 570 to $680 \mathrm{~nm}$. Such broad band was used to ensure probing the Purcell effect of QD emission in elliptical, ENZ, and hyperbolic regions of the multilayer metamaterial nanostructures ${ }^{112}$. A large PDOS in the ENZ and hyperbolic regions results in a higher Purcell factor and QD emission rate enhancement compared to the elliptical region. The measured photoluminescence decay data from QDs on the multilayer at emission wavelengths of 570 (ENZ region), 600, and $650 \mathrm{~nm}$ (hyperbolic region) show faster decay than that on the glass

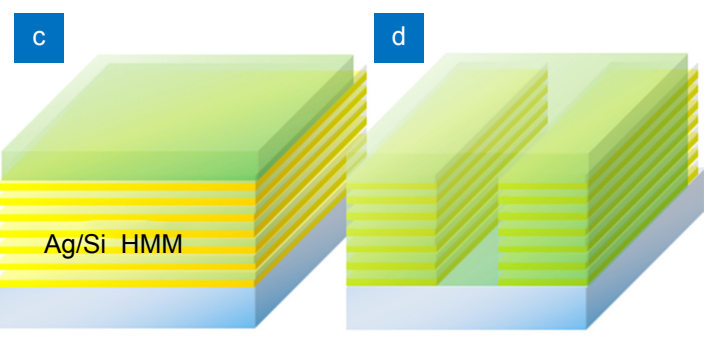

Fig. 6 | 1D grating out-couplers on multilayer HMMs. Schematic illustration of (a) Ag slab, (b) Ag grating, (c) Ag/Si HMM, and (d) Ag/Si HMM with grating. R6G fluorescence dye is in PMMA layers. Gratings period is $200 \mathrm{~nm}$. 


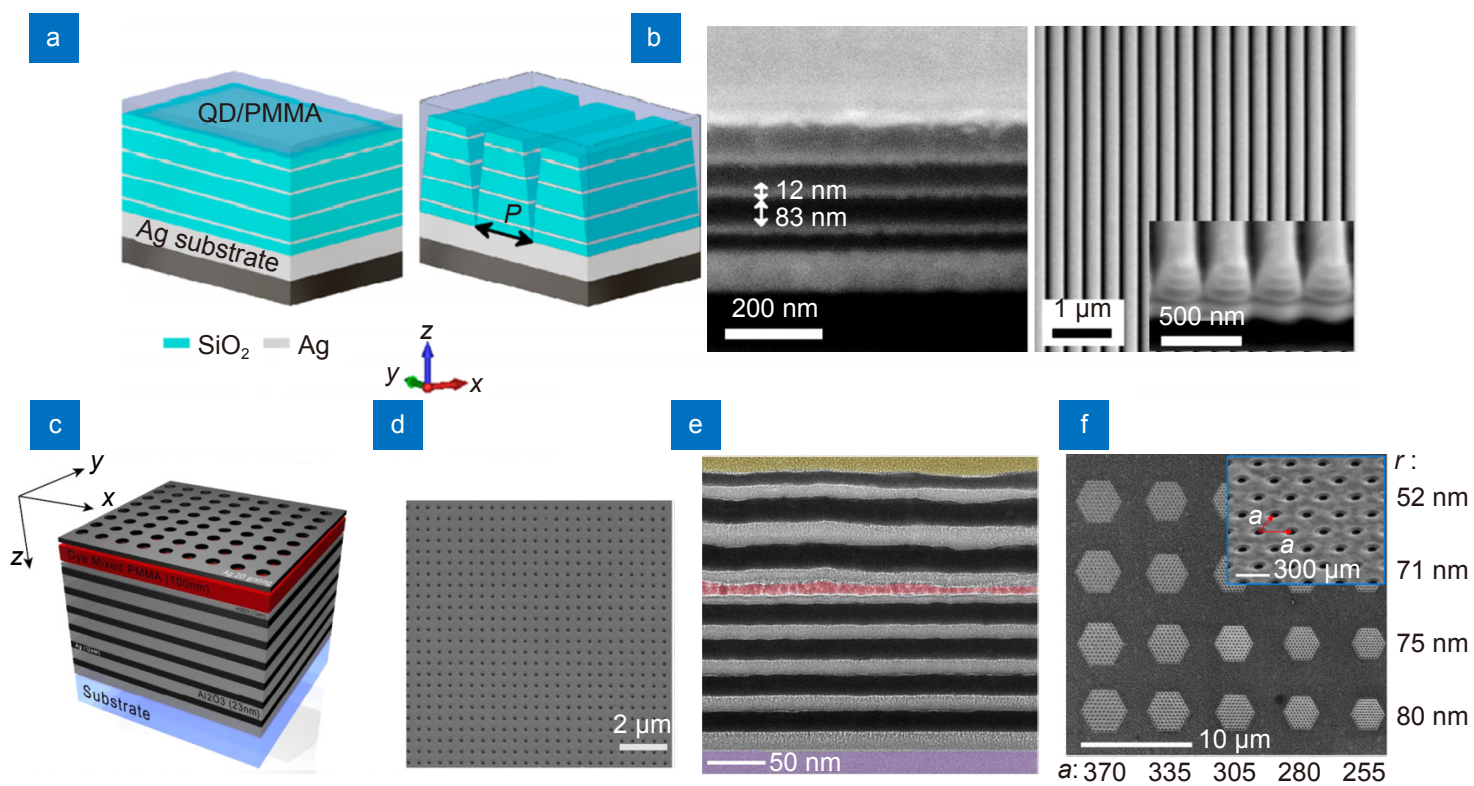

Fig. 7 | 1D and 2D grating out-couplers on multilayer HMMs. (a) Schematic illustration and (b) SEM images of HMM with 1D gratings. HMM is composed of four periods of $\mathrm{Ag}\left(12 \mathrm{~nm}\right.$ thick) and $\mathrm{SiO}_{2}(83 \mathrm{~nm})$ layers on top of $100 \mathrm{~nm}$ thick Ag substrate as a reflector. The pitch of grating is $P=$ $300 \mathrm{~nm}$. A thin layer of QDs in PMMA is spin-coated on top of the HMM structures. (c) Schematic of 2D grating in Ag film on HMM with 6 periods of $\mathrm{Ag}(12 \mathrm{~nm})$ and $\mathrm{Al}_{2} \mathrm{O}_{3}(23 \mathrm{~nm}$ ) layers. (d) SEM image of $2 \mathrm{D} \mathrm{Ag}$ on top of the PMMA layer with an average period of $500 \mathrm{~nm}$ and hole size of $160 \mathrm{~nm}$ (top view). (e) Cross-section view of transmission electron microscope (TEM) image of $\mathrm{HMM}$ made of $\mathrm{Ag}$ (dark color, $15 \mathrm{~nm}$ ), $\mathrm{Al}_{2} \mathrm{O}_{3}$ (bright color, $15 \mathrm{~nm}$ ), and embedded QD layer (false color). The bottom and top layers are glass substrate (false yellow) and Pt protection (false violet) layer, respectively (shown in false color). (f) SEM image of the top view of 2D gratings on HMMs with lattice constant of 255-370 nm with air hole radius of 52-80 nm. Figure reproduced with permission from: (a, b) ref. ${ }^{112}$, American Chemical Society; (c, d) ref. ${ }^{113}$, under a Creative Commons Attribution-NonCommercial- ShareAlike 4.0 International License; (e, f) ref. ${ }^{114}$, PNAS.

substrate. To further enhance the interactions, the multilayer HMMs was patterned as a grating with a pitch of $P=300 \mathrm{~nm}$ (see Fig. 7(a, b)). Such subwavelength grating provided larger spatial overlap and enabled stronger coupling between QDs inside the grating grooves and the high-k BPPs of the multilayer structure. A 6-fold emission rate enhancement by the multilayer grating structure was demonstrated in comparison with the reference case.

In addition, one-dimensional HMMs lattices were actively used for so-called "trapped rainbow" as a storage of electromagnetic waves. By changing the parameters of the plasmonic grating, trapping could be achieved for different wavelengths in a wide spectral range ${ }^{115,116}$. For example, in the 2014 work of the group F. J. Bartoli, the fabricated series of $1 \mathrm{D}$ nanopatterned gratings with different groove depths on a 300-nm-thick layer of Ag experimentally shows "trapped rainbow" effect in the $500-700$-nm region ${ }^{115}$. This effect is possible due to the accumulation of modes due to the rapid reduction of the SPP group velocity at specific position along the grating surface $^{115}$. HMMs support high-k modes with larger mode refractive index than plasmonic slabs for broader wavelength region, which propagate slower ${ }^{117}$ and the tapered HMM lattices or cavities were shown to trap electromagnetic modes and enhance light absorption for mid-infrared wavelengths ${ }^{118}$.

\section{Square or hexagonal lattice grating out-coupler}

By using a square lattice of air holes as a grating coupler on multilayer HMMs, the large spontaneous emission rate enhancement of an organic dye was demonstrated as illustrated in Fig. $7(\mathrm{c}, \mathrm{d})^{113}$. The metamaterial contains 12 alternating layers of $\mathrm{Al}_{2} \mathrm{O}_{3}$ and $\mathrm{Ag}$ (6 periods) with 23 $\mathrm{nm}$ and $12 \mathrm{~nm}$ thicknesses, respectively. To investigate the influence of the designed HMM on spontaneous emission enhancement, a dye doped PMMA layer (thickness around $100 \mathrm{~nm}$ ) was spin coated over a pre-deposited $\mathrm{Al}_{2} \mathrm{O}_{3}$ spacer $(12 \mathrm{~nm})$ on the HMM structure. Then the 2D grating with $500 \mathrm{~nm}$ period and $160 \mathrm{~nm}$ diameter air holes array was patterned above the dye mixed PMMA layer over the HMM, followed by the deposition of an $\mathrm{Ag}$ layer of thickness $20 \mathrm{~nm}$. The fluorescence time decay in spectral regions with different dispersion, such as elliptical dispersion of the HMM $(\lambda=420 \mathrm{~nm})$, the critical wavelength with the epsilon-near-zero regime for 
ordinary permittivity $(\lambda=430 \mathrm{~nm})$, and type II hyperbolic dispersion $(\lambda=450 \mathrm{~nm})$ were compared. Four different samples such as a reference (pink curve), HMM (red curve), HMM with Ag film on top (black curve) and HMM with a 2D grating (blue curve) were investigated for comparison. Among these configurations, a maximum of 18-fold decay rate enhancement is obtained for the grating coupled HMM structures at $510 \mathrm{~nm}$ emission wavelength (in the hyperbolic region), while the life time value is larger in the elliptical region.

As a follow-up work to further enhance emission, dye molecules were not deposited on top of the HMM, but directly embedded inside its volume, so that the emitters can interact more effectively with the BPP modes ${ }^{119}$. In order to compare the decay rate enhancement, three structures were investigated: a reference sample that is a spin coated DCM dye-dissolved $100 \mathrm{~nm}$ thick PMMA layer on a $5 \mathrm{~nm}$ thick $\mathrm{SiO}_{2}$ spacer on a glass substrate, the same thickness PMMA layer with dye on top of the HMM, and a $15 \mathrm{~nm}$ thick PMM layer with dye embedded inside the HMM. The dye molecules embedded in the HMM exhibit about a 35 -fold decay rate enhancement with respect to those in the reference sample. In addition, a 17-fold decay rate enhancement was obtained for the dye-dissolved PMMA layer on top of the HMM. This large spontaneous emission rate enhancement of the grating coupled HMM is attributed to the out-coupling of non-radiative plasmonic modes, as well as strong plasmon-exciton coupling in HMM via diffraction grating.

Broadband enhancement of spontaneous emission from two-dimensional semiconductors was demonstrated for molybdenum disulfide $\left(\mathrm{MoS}_{2}\right)$ and tungsten disulfide $\left(\mathrm{WS}_{2}\right)$ by placing the monolayers on a multilayer HMM with a grating coupler in hexagonal arrangement $^{120}$. Great advantage of using $2 \mathrm{D}$ materials is that the monolayer can be positioned at a very precise distance to the substrate to achieve the maximal enhancement effect of the PDOS. The HMM consisted of alternating layers of $\mathrm{Al}_{2} \mathrm{O}_{3}$ of about $20 \mathrm{~nm}$ thickness and $\mathrm{Ag}$ of about 10 $\mathrm{nm}$ thickness. For each Ag layer, an ultrathin germanium seed layer $(\sim 2 \mathrm{~nm})$ is first deposited that allows the silver to form optically smooth, high quality films. Photoluminescence measurements showed 56 times and 60 times intensity enhancement for the $\mathrm{WS}_{2}$ and $\mathrm{MoS}_{2}$, respectively, in comparison with the reference sample.

A multilayer HMM with a hexagonal array of built-in air holes was realized in order to extract enhanced emis- sion from quantum dots embedded in the HMM as shown in Fig. $7(\mathrm{e}, \mathrm{f})^{114}$. The HMM consists of 7 periods of $\mathrm{Ag}$ and $\mathrm{Al}_{2} \mathrm{O}_{3}$ with approximately $15 \mathrm{~nm}$ for both layers with $1 \mathrm{~nm}$ Ge adhesion layers at each interface of $\mathrm{Ag}$ and $\mathrm{Al}_{2} \mathrm{O}_{3}$. Quantum dots are colloidal CdSe/ZnS particles embedded inside the fifth from the substrate $\mathrm{Al}_{2} \mathrm{O}_{3}$ layer with the emission peak around $630 \mathrm{~nm}$. The grating is made of $120-160 \mathrm{~nm}$ diameter air holes arranged in a hexagonal lattice with period of $280-300 \mathrm{~nm}$. Twenty times enhanced spontaneous emission rate and one hundred times improved out-coupling efficiency of emission from quantum dots embedded in the structure was reported.

\section{Circular (bullseye) grating out-coupler}

A circular or bullseye grating is composed of concentric circular gratings with certain periods, so that the outcoupling of optical modes is achieved for every direction in the plane, where the grating is placed. The circular gratings have been employed for efficient out-coupling of enhanced photoluminescence from HMM structures too. The multilayer $\mathrm{Ag} / \mathrm{TiO}_{2}$ structure with a bullseye grating on top was theoretically studied ${ }^{121}$. The gratingHMM configuration exhibited 6-fold far-field Purcell factor. The spectral location of the out-coupled peak can be tuned by varying the bullseye grating period.

In another study, bullseye gratings with various pitches were created on top of a multilayer HMM made of $\mathrm{Al}_{2} \mathrm{O}_{3}(20 \mathrm{~nm})$ and $\mathrm{Ag}(12 \mathrm{~nm})$ layers with a thin $(1-2$ $\mathrm{nm})$ germanium $(\mathrm{Ge})$ adhesion layer between them ${ }^{122}$. Bullseye gratings with various half-periods, $\Delta=125,150$, $170,200,250,265$, and $300 \mathrm{~nm}$ were patterned on PMMA placed on top of the HMM. In order to enhance spontaneous emission from QDs by high-k BPP modes, QDs were embedded in the middle of HMMs by spincoating, for instance, in the $3^{\text {rd }}$ period out of 7 periods. The photoluminescence and lifetime of the patterned HMM were measured simultaneously using a confocal microscope. Measurements revealed that lifetime of QDs is decreased with increasing number of periods, indicating the presence of high-k modes and their contribution to the spontaneous emission enhancement. Observed lifetime for QDs located under the bullseye grating ( $\approx 1.9 \mathrm{~ns})$ was less than lifetime of QDs located away from the bullseye $(\approx 2.6 \mathrm{~ns})$.

A bullseye grating on a metal nanowire HMM was also studied theoretically ${ }^{123}$. A dipole emitter is placed on top of the $\mathrm{Au}$ nanowire HMM and a Si circular grating 
out-coupler is placed below the HMM structure. The enhanced emission is coupled to high-k BPP waveguide in the HMM slab and emitted to far-field via the bullseye grating. By optimizing the HMM slab thickness and fill ratio of metal nanowires, 18-fold far-field emission enhancement was predicted.

The summary of grating out-couplers performance is given in Table 3.

\section{Epsilon-near-zero (ENZ) materials}

An epsilon-near-zero (ENZ) material is an optical material whose relative permittivity (dielectric function) becomes near zero $(\varepsilon \sim 0)$ for a certain wavelength range ${ }^{124}$. Nanostructures, whose effective refractive index approaches the near zero regime are often referred to as near-zero-index (NZI) materials ${ }^{125}$. A thin slab of ENZ material (thickness $<\lambda / 50$, one fiftieth of the wavelength) supports a highly localized within-the-slab optical mode, sometimes referred to as an ENZ mode, enabling to concentrate light energy at nanoscale ${ }^{126,127}$. Recently, ENZ modes have been observed in a thin film of doped semiconductor, aluminum nitride $(\mathrm{AlN})^{127}$ and silicon carbide $(\mathrm{SiC})$ grating structures ${ }^{128}$ for mid-infrared wavelengths. Near the ENZ regimes, the hyperbolic (or indefinite) iso-frequency surfaces of HMMs changes its dispersion shape and allow wavevectors of BPPs up to infinitely high values (high-k modes regime) ${ }^{129-131}$. Thus, a high value of the PDOS in the vicinity of the ENZ wavelength can occur ${ }^{33,54,113,132,133}$. Consequently, if a fluorophore is placed near the ENZ medium, it experiences the enhanced Purcell effect ${ }^{32,33,132,134}$.

Planar plasmonic multilayers as illustrated in Fig. 8(a, b) were designed to exhibit single ENZ (permittivity becomes near zero) and double ENZ behavior (both permittivity and permeability simultaneously become near zero) ${ }^{135}$. The single ENZ structures consist of metal-insulator (dielectric)-metal (MIM) layers with $20 \mathrm{~nm}$ thick Ag layers and $80 \mathrm{~nm}$ thick $\mathrm{Al}_{2} \mathrm{O}_{3}$ layers. The first ENZ condition, which occurs at shorter wavelengths, is tuned to match the absorbance band of the fluorophore placed on the top of MIM structures with $\mathrm{Al}_{2} \mathrm{O}_{3}$ spacer separation. Cesium lead halide perovskite $\left(\mathrm{CsPbBr}_{3}\right)$ nanocrystals are used as a fluorophore that emits in the green spectral region. The double ENZ structures are composed of MIMIM configuration with the same metal and dielectric layers, such that ENZ wavelengths are also tuned to the emission peak of the fluorophore. The maximum PL enhancement occurs for a space layer thickness of $50 \mathrm{~nm}$ [see the inset of Fig. 8(c)]. In order to highlight the contribution of both ENZ conditions, a comparison between the single ENZ regime in the absorption band of the fluorophore and double ENZ case

Table 3 | Summary of photoluminescence enhancement by grating out-coupler on HMM structures. Unless noted, emitters are located on the top surface of HMM structures and the works are experimental. Here $P$ is the pitch or lattice constant of the grating out-coupler.

\begin{tabular}{|c|c|c|c|}
\hline HMM structures and materials & $\begin{array}{l}\text { Emitters (emission peak } \\
\text { wavelength) }\end{array}$ & Enhancement factor & $\begin{array}{c}\text { References } \\
\text { (year) }\end{array}$ \\
\hline 1D grating $(P=200 \mathrm{~nm})$ on $\mathrm{Ag}(9 \mathrm{~nm}) / \mathrm{Si}(10 \mathrm{~nm}), 15$ period & R6G dye $(600 \mathrm{~nm})$ & 80 & ref. ${ }^{110}(2014)$ \\
\hline 1D grating $(P=300 \mathrm{~nm})$ on $\mathrm{Ag}(12 \mathrm{~nm}) / \mathrm{SiO}_{2}(83 \mathrm{~nm}), 4$ period & $\begin{array}{l}\text { CdSe/ZnS QDs } \\
(570-680 \mathrm{~nm})\end{array}$ & 6 & ref. ${ }^{112}(2017)$ \\
\hline $\begin{array}{l}\text { 1D grating }(P=200 \mathrm{~nm}) \text { on } \mathrm{Ag}(10 \mathrm{~nm}, 10 \text { layer }) / \\
\qquad \mathrm{Si}(10 \mathrm{~nm}, 11 \text { layers })\end{array}$ & Dipole emitters (582 nm) & 120 (Theory) & ref. ${ }^{111}(2018)$ \\
\hline $\begin{array}{l}\text { Square lattice grating }(P=500 \mathrm{~nm}) \text { on } \mathrm{Ag}(12 \mathrm{~nm}) / \mathrm{Al}_{2} \mathrm{O}_{3}(23 \mathrm{~nm}) \text {, } \\
6 \text { period }\end{array}$ & Coumarin $500(510 \mathrm{~nm})$ & 18 & ref. ${ }^{113}(2014)$ \\
\hline $\begin{array}{l}\text { Dye molecules embedded grating-coupled } \mathrm{HMM}(\mathrm{GC}-\mathrm{DEHMM}) \\
\qquad(P=500 \mathrm{~nm} \text { and hole radius } 100 \mathrm{~nm}) \text { on } \\
\mathrm{Ag}(12 \mathrm{~nm}) / \mathrm{SiO}_{2}(5 \mathrm{~nm}) / \text { dye-dissolved PMMA }(15 \mathrm{~nm})\end{array}$ & DCM dye $(580 \mathrm{~nm})$ & $\begin{array}{c}35 \text { for the GC-DEHMM, } 17 \text { GC- } \\
\text { DEHMM with respect to the } \\
\text { DEHMM }\end{array}$ & ref. $^{119}(2016)$ \\
\hline $\begin{array}{l}\text { Hypercrystal, hexagonal lattice grating }(P=280 \mathrm{~nm} \text { and hole } \\
\text { radius } 100 \mathrm{~nm}) \text { on } \mathrm{Ag}(20 \mathrm{~nm}) / \mathrm{Al}_{2} \mathrm{O}_{3}(20 \mathrm{~nm})\end{array}$ & $\begin{array}{c}\mathrm{MoS}_{2}(660 \mathrm{~nm}) \mathrm{WS}_{2} \\
(620 \mathrm{~nm})\end{array}$ & $\begin{array}{l}56 \text { times enhancement for } \mathrm{WS}_{2}, 60 \\
\text { times for } \mathrm{MoS}_{2}\end{array}$ & ref. $^{120}(2016)$ \\
\hline $\begin{array}{l}\text { Hypercrystal, hexagonal lattice grating }(P=280-300 \mathrm{~nm}) \text { on } \\
\qquad \mathrm{Ag}(15 \mathrm{~nm}) / \mathrm{Al}_{2} \mathrm{O}_{3}(15 \mathrm{~nm}) \text { multilayer, } 7 \text { period }\end{array}$ & CdSe/ZnS QDs (630 nm) & 20 times & ref. ${ }^{114}(2017)$ \\
\hline $\begin{array}{l}\text { Bullseye grating }(P=400 \mathrm{~nm}) \text { on multilayer HMM Ag } \\
\qquad(10 \mathrm{~nm}) / \mathrm{TiO}_{2}(30 \mathrm{~nm}), 4.5 \text { period }\end{array}$ & Emitter $(800 \mathrm{~nm})$ & 6 far-field Purcel factor (Theory) & ref. $^{121}(2013)$ \\
\hline $\begin{array}{c}\text { Bullseye grating }(P=250-600 \mathrm{~nm}) \text { on } \mathrm{Ag}(12 \mathrm{~nm}) / \mathrm{Al}_{2} \mathrm{O}_{3}(20 \mathrm{~nm}) \\
\text { multilayer }\end{array}$ & $\begin{array}{l}\text { CdSe/ZnS quantum dots } \\
\qquad(630 \mathrm{~nm})\end{array}$ & 20 & ref. $^{122}(2015)$ \\
\hline $\begin{array}{l}\text { Si bullseye grating ( } P=600 \mathrm{~nm}) \text { on Au nanowire ( } 44 \mathrm{~nm} \text { pitch }) \text { in } \\
\text { PMMA HMM (100 nm thickness) }\end{array}$ & Dye $(850 \mathrm{~nm})$ & 18 (Theory) & ref. $^{123}(2019)$ \\
\hline
\end{tabular}



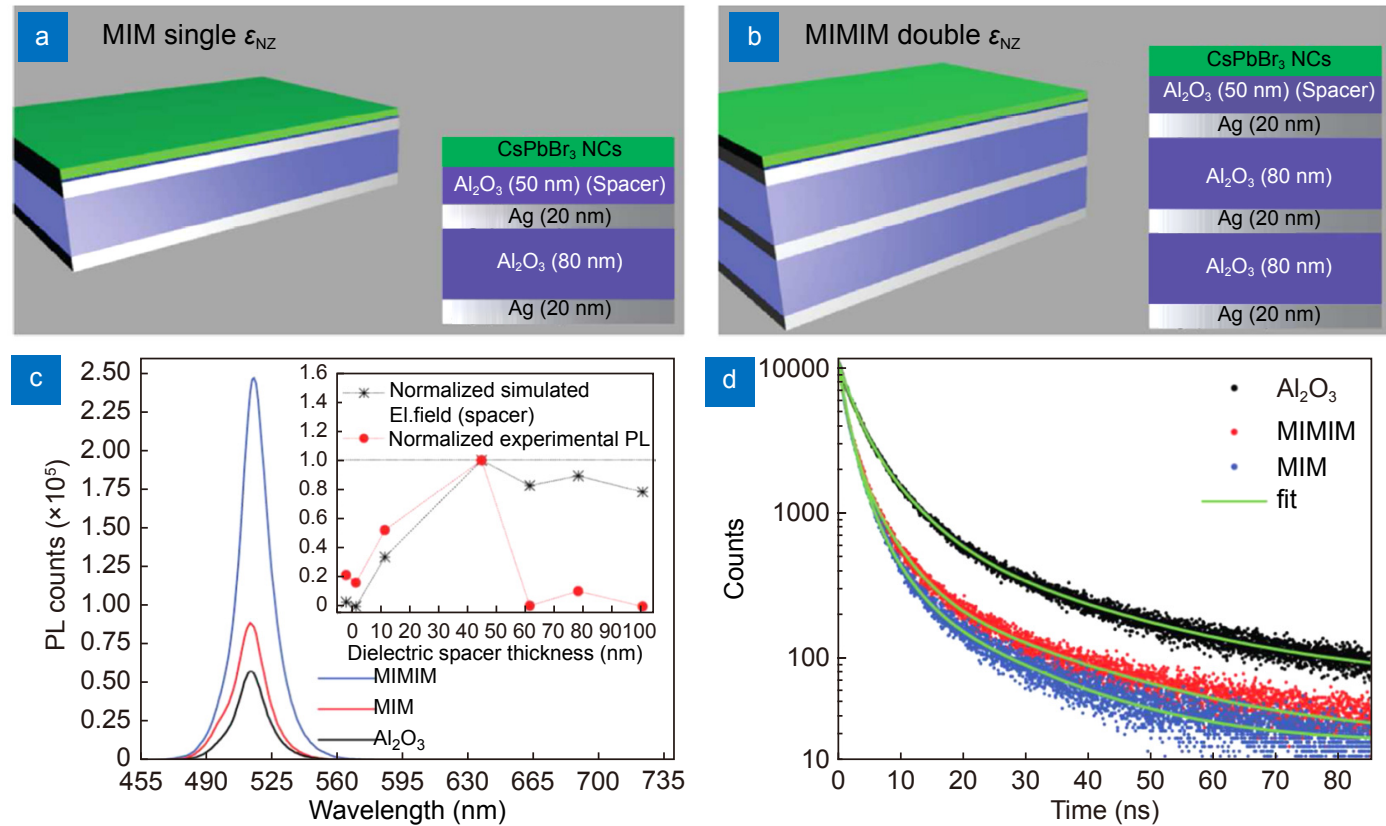

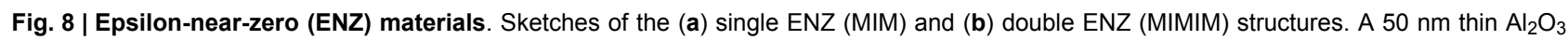
layer has been deposited on top of each structure as a spacer between the dye ( $\mathrm{CsPbr}_{3}$ nanocrystals) and the $\mathrm{Ag}$ layer. (c) Spontaneous emission and decay times of $\mathrm{CsPbBr}_{3}$ nanocubes deposited on a bare $\mathrm{Al}_{2} \mathrm{O}_{3}$ substrate (black), a MIM (red), and a MIMIM (blue) structure 1D grating. (d) PL enhancement by plasmonic nanostructures. Figure reproduced with permission from ref. ${ }^{135}$, American Chemical Society.

was presented. In the case of the single ENZ, small increase in the emission (by about a factor of 1.5 compared with a bare $\mathrm{Al}_{2} \mathrm{O}_{3}$ layer) and a slightly reduced decay time was observed, while a 4 -fold enhancement of the emission, accompanied by a significantly shortened decay time, was observed for the double ENZ case as shown in Fig. 8(c, d). In addition, double ENZ wavelengths for both near zero permittivity and permeability wavelengths can be tuned within the whole visible range by adjusting the thickness of each single dielectric layer in order to use a wide variety of fluorophores.

\section{Active HMMs}

As shown above, layered metal-dielectric HMMs support a wide variety of surface and BPP modes with high modal confinement inside the multilayer. Far-field radiation can excite only a subset of these polaritonic modes, typically with a limited energy and momentum range in respect to the wide set of large wave vectors (high-k modes) of BPP modes supported by hyperbolic dispersion media. Localization of light emitters (dye molecules, quantum dots, and nanodiamonds with color centers) in the HMMs' bulk makes it possible to excite many BPP modes directly. The relaxation of the excitation of these modes, accompanied by radiation, provides tools for creating light sources with a controlled spectral composi- tion determined by the resonance properties of the HMMs.

Of particular practical interest is the creation of light sources characterized by a spectrally narrow emission, since the high spectral brightness realized in such sources is fundamentally important in molecular spectroscopy and related sensing with a sensitivity at the level of single molecules ${ }^{136,137}$, and future electronics operating at optical frequencies. ${ }^{138,139}$ However, optical losses in metals do not allow achieving high- $\mathrm{Q}$ resonance properties of existing metamaterials and plasmonic devices. Thus, the characteristic values of the spectral width of the corresponding resonances are at the level of 30-50 $\mathrm{nm}$ in the visible spectral range. Loss compensation (transition from passive to active systems) can overcome this limitation. The inclusion of gain media offers a pathway to mitigate these losses, and gives rise to the new class of active HMMs, where light strongly interacts with quantum transitions of the active medium ${ }^{140-142}$. In active HMMs the simultaneous interaction of light with an absorption and emission lines of optically pumped gain media can take place. The use of stimulated emission and loss compensation are necessary ingredients for achieving the lasing regime and have been known in laser physics since the invention of the first laser. However, the use of small optical cavities significantly complicates the 
physical picture of lasing. Indeed, to achieve loss compensation for a HMM cavity, it is necessary to have a large number of emitters in the high-intensity region of the respective mode ${ }^{143}$. However, the small mode volume of such resonators containing a high number of emitters leads to hybridization of quantum emitters with the HMM cavity-an effect very difficult to achieve in photonic lasers due to the significantly large dimensions of such resonators, in comparison with the optical wavelength $^{144}$.

Figure 9 shows two typical schemes for creating an active HMM: with dye molecules located on top of the HMM (Fig. 9(a)) and dye molecules located in each dielectric layer of the HMM (Fig. 9(b)). The first scheme is simpler in experimental implementation, while the second one provides the highest saturation of the BPP modes volume by dye molecules. The energy levels of the dye molecules that provide gain can be approximated by a four-level model as sketched in Fig. 9(c). Pumping of the dye and population inversion involves the excitation of electrons from the fundamental level to the excited state (blue arrow in Fig. 9(c)). The electrons relax through fast non-radiative processes (green arrows in Fig. 9(c)) and spontaneous and stimulated light emission occurs via the optically active transition from $E_{3}$ to $E_{4}$ providing gain (red arrow in Fig. 9(b)). The choice of the four-level system for active layer is important to get great population inversion (population of $E_{3}$ energy level compared to $E_{4}$ level) and hence high gain. To obtain a physical pattern of a system presented in Fig. 9, full quantum analysis of coupled electronic-vibronic system is required, involving understanding of the absorption, emission, and plasmon tunings, together with phonon and relaxation pathways, while also exploring the effect of the tight plasmonic confinement on the molecular relaxation. Although there have been some recent progress in developing models ${ }^{145-147}$, a full theory accounting for how different phonon subsystem interact with the optical cavity is currently missing for purely dielectric cavities, plasmonic cavities formed by nanostructures and for HMMs, as well.

Arrangement of emitters in the field of an optical cavity can modify the spontaneous emission rate leading to the reduced lifetime and altered far-field emission pattern ${ }^{150}$. Two regimes of emitter-optical cavity interactions are known: (i) weak-coupling ${ }^{151-153}$ and (ii) strongcoupling regime ${ }^{154-156}$. Strong coupling, unlike the weakcoupling regime, relies on the back action between the emitter and metamaterial to create coherent states between light and matter. In the weak coupling regime considered in the previous sections, only a small perturbation of the band takes place. In the strong coupling regime, both emission and absorption lines produce extreme distortions of the plasmonic band due to Rabi splitting and a parity-time (PT) symmetry broken phase with generation of exceptional points at the loss-gain compensation frequencies ${ }^{147,149}$. It is important to point out that in the strong coupling regime the photonic and excitonic components of the system cannot be treated as separate entities, as they form new polaritonic eigenstates (exciton-polaritons) having both light and matter characteristics.

To commit optical pumping and light-injection into hyperbolic modes the dye-molecules energy levels should match BPP bands. HMMs have distinct plasmonic bands, the lower ones providing the propagation of extremely high momentum waves and the upper ones lying partially inside the light cone. The absorption line of the dye should fall within the upper bands inside the light cone, while the emission line should reside in the lower bands. This particular arrangement allows for light emission into the hyperbolic modes, benefiting simultaneously from both strong field enhancement and large wavevectors. To describe weak and strong coupling scenarios in active HMMs, we will follow in the following
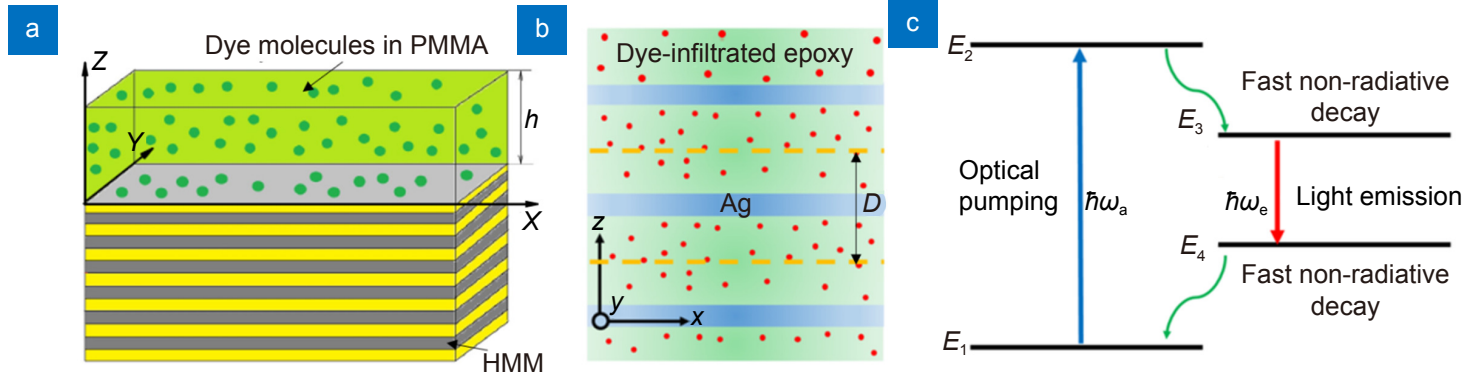

Fig. 9 | Active HMM structure: (a) dye molecules mixed in PMMA located on top of HMM; (b) dye molecules arranged in dielectric layers of HMM; (c) energy diagram of a four-level dye molecule, with an absorption (blue) and emission line (red). Figure reproduced with permission from: (a) ref. ${ }^{148}$, American Physical Society; (b) ref. ${ }^{149}$, American Chemical Society. 
discussion an analytical approach developed in an article $^{149}$. A simplified analysis of active HMMs considers coupling with only one excitonic line (either the emission or absorption line). In this case a semi-classical model for this system can be found from the MaxwellBloch equations. The result can be written in the form of the coupled oscillator equations for the modal field amplitude $E$ and the polarization of the respective transition $P$ :

$$
\begin{aligned}
\partial_{\mathrm{t}}\left(\begin{array}{c}
E \\
P / \varepsilon_{0}
\end{array}\right) & =-\mathrm{i} \Omega_{ \pm}\left(\begin{array}{c}
E \\
P / \varepsilon_{0}
\end{array}\right) \\
& =\left(\begin{array}{cc}
-\mathrm{i} \Omega_{\mathrm{m}} & \mathrm{i} A_{\mathrm{m}} \\
\mathrm{i} K_{12} & -\mathrm{i} \Omega_{12}
\end{array}\right)\left(\begin{array}{c}
E \\
P / \varepsilon_{0}
\end{array}\right),
\end{aligned}
$$

where $\Omega_{\mathrm{m}}$ and $\Omega_{12}$ are the complex frequencies of the selected optical mode and the excitonic transition (absorption or emission), whose negative imaginary parts are the modal damping rate $\gamma_{\mathrm{m}}$ and the linewidth of the transition $\left(\gamma_{\mathrm{a}}\right.$ or $\left.\gamma_{\mathrm{e}}\right)$, respectively. The off-diagonal coupling terms are determined as $A_{\mathrm{m}}=\Omega_{\mathrm{m}} \Gamma / 2$ and $K_{12}=\sigma_{12} / \varepsilon_{0}$, introducing the spatial overlap factor $\Gamma$ of the plasmonic mode with the dielectric medium, and the cross-section $\sigma_{12}$ of the excitonic transition under consideration.

Solving for the eigenfrequencies $\Omega_{ \pm}$of the coupled system (8) defines the mode splitting within the semi-classical coupling model for two oscillators:

$$
\Omega_{ \pm}=\frac{1}{2}\left(\Omega_{\mathrm{m}}+\Omega_{12}\right) \pm \frac{1}{2} \sqrt{4 K_{12} A_{\mathrm{m}}+\left(\Omega_{\mathrm{m}}-\Omega_{12}\right)^{2}} .
$$

It is important to note that, with all other parameters determined, the only remaining parameters to fit are the frequency of the selected optical mode $\Omega_{\mathrm{m}}$, and its spatial overlap factor $\Gamma$ with the gain layers. Both parameters depend only on properties of the passive structure and are independent of the dye characteristics. The cross-section $\sigma_{12}$ is related to the population levels via

$$
\sigma_{12}=-\frac{\mu_{12}^{2}}{3 \hbar}\left(N_{2}-N_{1}\right)
$$

where $\mu_{12}$ is the dipole matrix element, $N_{1}$ and $N_{2}$ are the density of atoms in the ground and excited states, respectively. For absorption transitions (where $N_{2}<N_{1}$ ), Eq. (9) describes the usual transition from weak coupling to strong coupling, manifesting itself as vacuum Rabi splitting within the semi-classical framework. As the cross-section becomes negative for emission processes $\left(N_{2}>N_{1}\right)$, the term $4 K_{12} A_{\mathrm{m}}$ in the discriminant of Eq. (9) becomes negative, as well. For sufficiently large coupling, the sign flips, and the emission line produces a splitting behavior similar to the fork observed in PT symmetry breaking scenarios. In the complete case of four-level dyes, coupling occurs simultaneously to both emission and absorption lines. Each line provides its own polarization that interacts with the amplitude of the optical mode. This results in a modified coupling model.

It is possible to distinguish two considered regimes (a weak coupling regime characterized by small distortions of the dispersion, and a strong coupling regime leading to Rabi splitting of modes) in light reflectance from a HMM sample. Note the weak or strong coupling regimes can be realized through the dye molecules - HMM modes overlapping factor $\Gamma$. Figure 10(a) shows the reflectance map of a finite structure presented at Fig. 9(b) formed by 10-unit cells (a single unit cell is marked by the orange dashed lines in the figure) via transfer-matrix calculations, with each reflectance maximum indicating a discrete plasmonic mode. For a small value of coupling a slight perturbation and smearing of the optical modes is observed around $\omega_{\mathrm{a}}=0.313 \omega_{\mathrm{p}}$ (an indication of a weak coupling regime) when compared with the passive case.

The dispersion is qualitatively changed in the strong coupling regime (Fig. 10(b)). In this case anti-crossings appear, and a gap opens in the reflectance map. This effect is particularly visible for the discrete system of modes in finite structures. In the figure, two such modes obtained with the use of the semiclassical model Eq. (9) are marked with the green and magenta curves. From Fig. 10(b) it is obvious that close to the crossing point of the optical mode and the excitonic line $\left(\omega_{\mathrm{m}}=\omega_{\mathrm{a}}\right)$ the first term in the square root of Eq. (9) dominates. In the strong coupling regime the resonance linewidth of the optical mode and the exciton are smaller than the expected splitting, the square root becomes real and a gap opens. The width of this gap is the Rabi splitting energy $\hbar \Omega_{\text {Rabi }}=\sqrt{2 K_{\mathrm{a}} A_{\mathrm{m}}}$ equals about $0.02 \hbar \omega_{\mathrm{p}}$. Figure 10(c) shows the dispersion of the polaritons calculated with use of Eq. (9) (green curves) using parameters of the excitonic line (blue dashed curve) and the unperturbed optical mode (black dashed curve). Away from the crossing, the polaritons simply follow the two components, with one polariton being dominantly plasmonic (polariton 1) while the other is dominantly excitonic (polariton 2) for small wavevector $k_{x}$ (and vice versa for large $k_{x}$ ). At the crossing point the gap opens, and the two polaritons form a mixed state between the excitonic and plasmonic modes.

The full system presented by four-level dye molecules embedded in the dielectric medium of multilayer HMMs with impact of coupling on both absorption and 

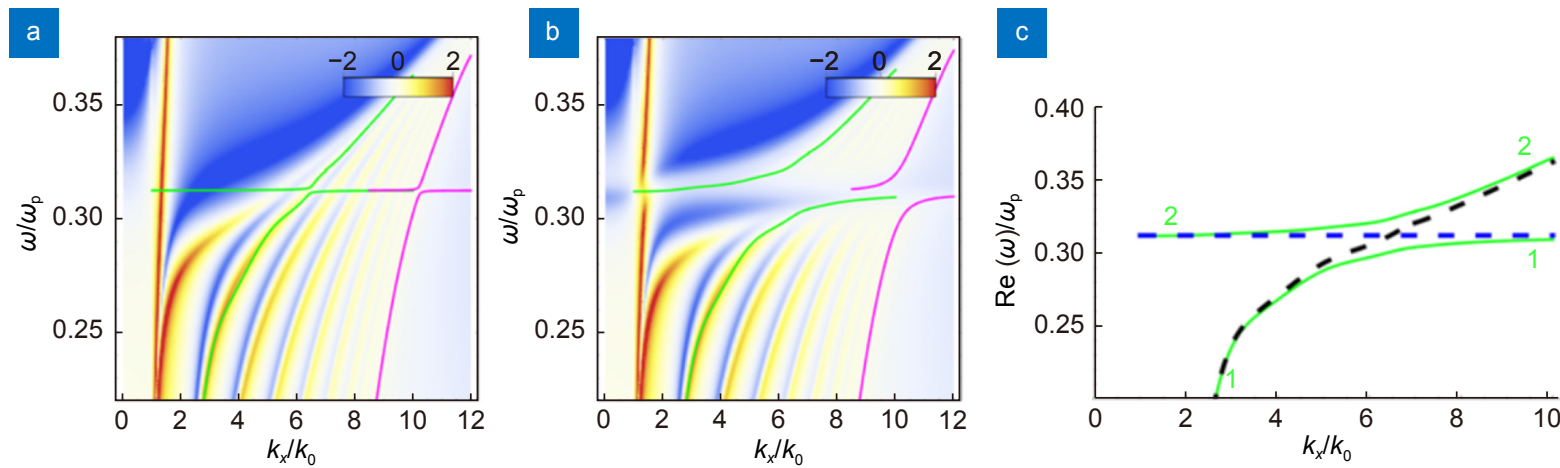

Fig. 10 | FDTD simulations and analytical calculations of emitter-HMM coupling in light reflectance: (a) reflectance with an absorption line in the weak coupling regime, (b) strong coupling regime for the finite structure of HMM Fig. 9(b) (logarithmic scale), (c) real and imaginary parts of the complex eigenfrequencies $\Omega_{ \pm}$obtained with the model (green curves) in the strong coupling regime. The green and magenta curves are obtained via semi-classical model for two different modes (Eq. (9)). The blue dashed curve is the excitonic absorption line and black dashed curve is the unperturbed optical mode. The two polaritons resulting from the coupling of the optical mode with the excitonic line are identified by numbers 1 and 2 ( $\Omega_{-}$and $\Omega_{+}$, respectively). Figure reproduced with permission from ref. ${ }^{149}$, American Chemical Society.

emission was further studied in paper $^{149}$. It was shown that introduction of gain in HMMs provides a tool for loss-compensation and opens a convenient route facilitating injection of light into hyperbolic modes. At the loss-gain compensation conditions both emission and absorption lines produce extreme distortions of the plasmonic band due to Rabi splitting and a PT-symmetry broken phase with generation of exceptional points. The presence of exceptional points has significant implications on the group index (along $z$ direction) $n_{g, z}$, Figure $11(\mathrm{a}, \mathrm{b}, \mathrm{c})$ show zoom-ins on two exceptional points, providing evidence that $n_{g, z}$ diverges, which is a signature of the existence of exceptional points, connecting with recent results ${ }^{157,158}$.

Recent studies show that the equal-frequency surface model suffers to describe the topology of entire bands for continuous HMMs. Better description can be found with use of a non-Hermitian Hamiltonian formed from Maxwell's equations. Successful implementation of this approach helped to find two types of three-dimensional non-Hermitian triply degenerate points with complex linear dispersions and topological charges \pm 2 and 0 induced by chiral and gyromagnetic effects, paving the way for exploring topological phases in photonic continua and device implementations of topological $\mathrm{HMMs}^{159}$. The effect of strong coupling on laser emission generation was further studied in many details, including effects of exceptional points and search for lowering down lasing threshold as well as narrowing corresponding radiation linewidth ${ }^{160}$.

Only few experimental works are known in this field. The first observation of strong coupling of emitters with HMM was reported by Indukuri et $\mathrm{al}^{109}$. A monolayer of quantum dots was arranged on top of HMM formed by
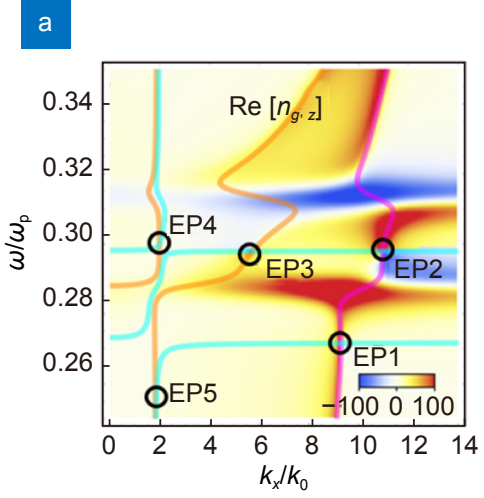
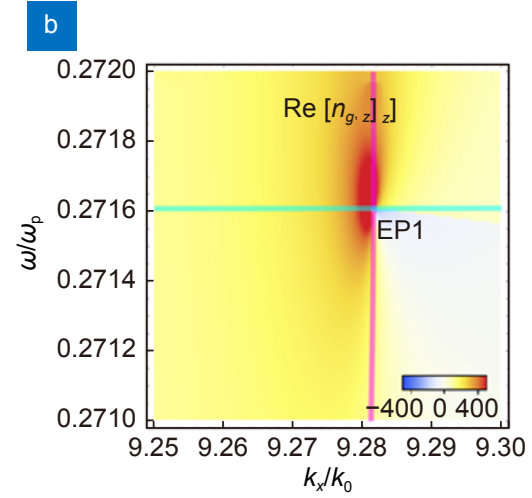

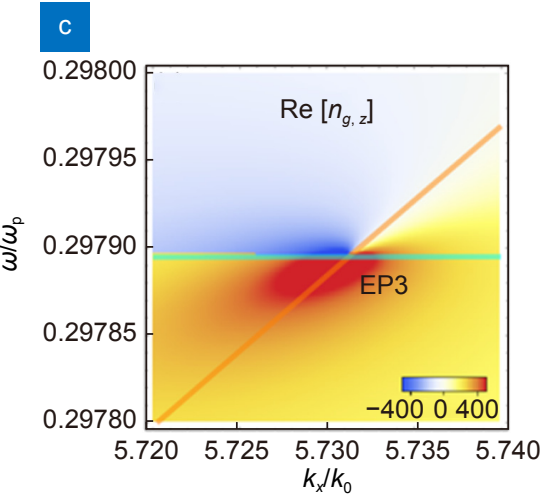

Fig. 11 | Exceptional points (EP) in the system formed by four-level dye molecules embedded in the dielectric medium of multilayer HMMs: (a) group index in $z$ direction $n_{g, z}$ of the dye infiltrated multilayer, (b) zoom on EP1, (c) zoom on EP3. Orange curves correspond to the band edges at the Brillouin zone center $k_{z} D=0$, magenta curves correspond to the band edges at the Brillouin zone edge $k_{z} D=\pi$, and cyan curves correspond to the gain-loss compensation line where $\operatorname{Im}\left(\cos \left(k_{z} D\right)\right)=0$. Figure reproduced with permission from: ref. ${ }^{149}$, American Chemical Society. 
Ag nanowires, Fig. 12(a), with a number of CdSe QDs per HMM unit cell equals about 350. Figure 12(b) shows photoluminescence spectra of the CdSe QD monolayer for various polymer spacer layer thickness $h$. Splitting of the photoluminescence peaks are clearly seen at small $h$ values only. This splitting corresponds to coupling energy of about $120 \mathrm{meV}$ and fits theoretical expectations. For large $h$, these peaks disappear due to reduced coupling of QDs to HMM modes. Note the ambiguities in the strong coupling evidence interpretation ${ }^{161}$. The demonstrated splitting in photoluminescence spectrum was not verified in scattering and can be related to excitation of dark modes of HMMs leading to a dip in the QDs photoluminescence, rather than associated with a strong coupling regime.

Stimulated emission of surface plasmons on top of metamaterials with hyperbolic dispersion as well as losses compensation were realized in a number of works. The very first experimental verification of stimulated emission of surface plasmons propagating on top of metal-dielectric multilayered HMMs coated with optically pumped dye-doped polymer for the wavelengths around $860 \mathrm{~nm}$, as well as loss compensation was reported in ref. ${ }^{81}$. The HMMs were composed of $\mathrm{Ag} / \mathrm{MgF}_{2}$ alternating layers. In the realized experimental conditions, the stimulated emission of propagating plasmons on top of the metamaterial was enhanced by the nonlocal dielectric environment and high local PDOS.

The loss compensation and lasing action in metamaterials based on gold nanorod arrays coated with thin films of PVA embedded with R101 dye was demonstrated $^{108}$. Depending on the chosen parameters (metal fill ratio), the sample under study can exhibit hyperbolic
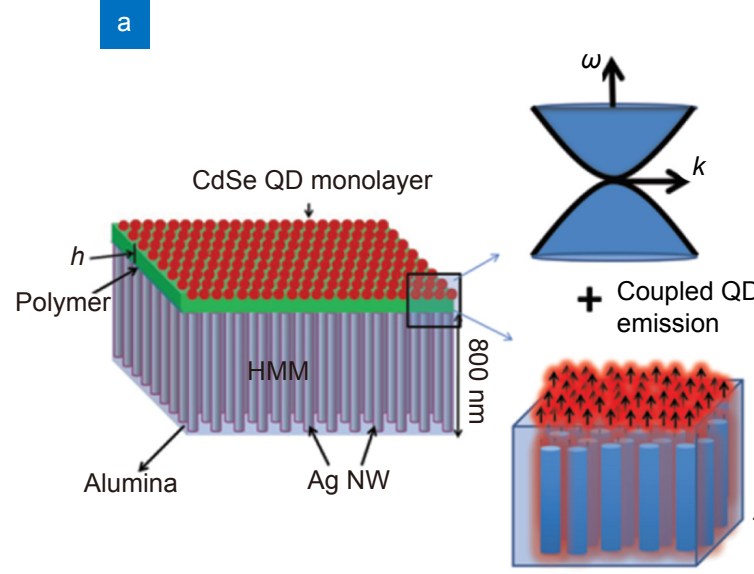

or elliptic dispersion. With both types of metamaterials, a lasing action was demonstrated with the emission line width as narrow as $6 \mathrm{~nm}$ when the samples were pumped above respective thresholds compared to more than 60 $\mathrm{nm}$ wide pristine spectrum of dye molecules.

The first successful attempt to demonstrate the integration of the HMM with random laser systems for enhancing stimulated emission and reducing lasing threshold was reported in ref. ${ }^{79}$. The sample design included $\mathrm{ZnO}$ nanoparticles as the active material arranged in a random order on top of HMM samples. The two kinds of $\mathrm{Ag} / \mathrm{MoO}_{3}$ multilayers with hyperbolic and elliptic dispersion, respectively, were fabricated. The first HMM sample exhibiting hyperbolic dispersion contained 6 pairs of $\mathrm{Ag}(22 \mathrm{~nm})$ and $\mathrm{MoO}_{3}(10 \mathrm{~nm})$ layers with the $\mathrm{Ag}$ fill-fraction of $68.75 \%$. As for the second sample with elliptic dispersion, the structure was composed of $\mathrm{Ag}(12 \mathrm{~nm})$ and $\mathrm{MoO}_{3}(20 \mathrm{~nm})$ layers with the Ag fill-fraction of $37.5 \%$. The random laser action was realized with $\mathrm{ZnO}$ nanoparticles on $8 \mathrm{~nm}$ thick $\mathrm{MoO}_{3}$ deposited as a capping spacer layer to prevent unwanted oxidation and quenching of the emission from $\mathrm{ZnO}$ nanoparticles. The first HMM structure showed about $20 \%$ reduction of lasing threshold and about 6 times higher emission intensity. The rough interface between $\mathrm{ZnO}$ nanoparticles and the HMM assisted the out-coupling of the high-k modes to the far-field rather than letting the emitted light get trapped inside the multilayer structure. The Purcell factor for the HMM sample at the wavelength of $395 \mathrm{~nm}$, which corresponds to the spontaneous emission of $\mathrm{ZnO}$ nanoparticles, is larger than that of the second sample with elliptic dispersion. Furthermore, deep-UV LED on a HMM system was b

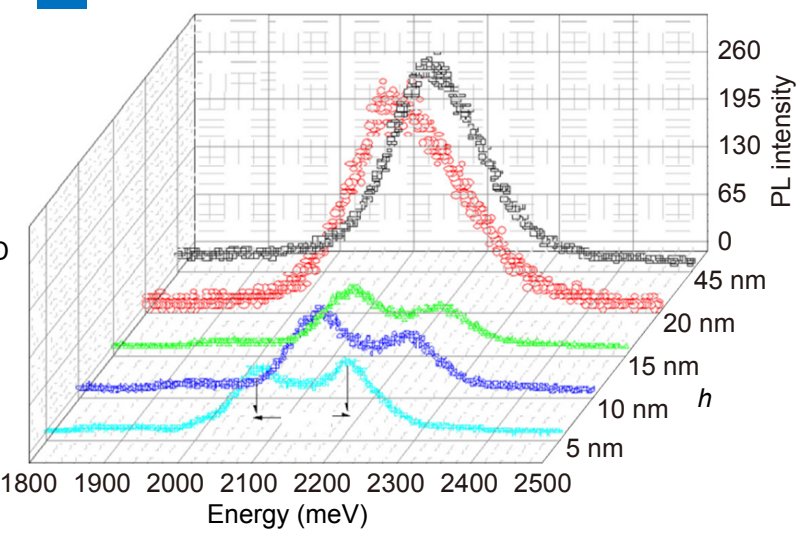

Fig. 12 | Coherent interaction between HMM and multiple emitters leading to strong coupling: (a) schematic diagram of HMM with QDs on top of the polymer spacer, (b) PL measurement on QD monolayer. The magnitude of the splitting of the various PL peaks are indicated by the quantity $\Delta E_{\text {exp. }}$ Figure reproduced with permission from ref. ${ }^{109}$, The Royal Society of Chemistry. 
realized $^{77}$. The LED was made with three pairs of AlGaN MQWs with a 318-nm emission wavelength and a 15-nm thick AlGaN cap layer grown on sapphire. The HMM consisted of four pairs of $\mathrm{Al}(20 \mathrm{~nm})$ and $\mathrm{MgF}_{2}(20 \mathrm{~nm})$ layers deposited on the LED surface. A 160-fold increase in the radiative emission rate and a 3.5 -fold increase in the quantum efficiency were achieved. Moreover, the capability of metacavity in tailoring the direction of light emission led to a $520 \%$ increase in total emission intensity and $148 \%$ increase in emission extraction.

In recent paper ${ }^{162}$, deep-ultraviolet lasing at room temperature using a hyperbolic meta-cavity and multiple quantum-well sample was demonstrated. The meta-cavity was formed by nanoscale HMM cubes with four pairs of $\mathrm{Al}$ and $\mathrm{MgF}_{2}$ alternating layers with $20 \mathrm{~nm}$ thickness each, dimensions of $200 \times 200 \mathrm{~nm}^{2}$, and a spacing between cubes of $200 \mathrm{~nm}$. The plasmon resonance mode merges within the cube and provides a resonant radiation feedback to the multiple quantum-well as active medium. The latter consists of AlGaN semiconductor with a wide bandgap (3.4-6.2 eV) in the UV range. The HMM high-k modes allow the dipoles with various orientations to contribute to radiative emission, achieving enhancement of spontaneous emission rate by a factor of 33 and quantum efficiency by a factor of 2.5. With pumping above a threshold, the sample shows a clear narrowing of emission line spectral width down to 0.8 $\mathrm{nm}$ at $289 \mathrm{~nm}$ emission wavelength.

This young direction of research is still in a rapid development. A number of successful results has been shown, however more research is anticipated in theory as well as in experiment. Deeper understanding the effect of light matter strong coupling for the case of HMMs cavities is of high demand, including the Raman scattering as well as statistics of corresponding emission ${ }^{163}$. Recent studies with other types of cavities show possibility of vibrational strong coupling in Raman scattering ${ }^{164}$. Getting any type of statistics of emitted photons ${ }^{165}$ suggests new areas for HMMs applications.

\section{Conclusion}

In this review, we have provided an overview of current progress in photoluminescence control on various types of HMMs and metasurfaces. Highly localized electric fields of high-k bulk plasmon polaritons enable large optical density of states and extreme anisotropy. The bandwidth of the hyperbolic region where high-k modes are supported is extremely broad for HMM, spreading from certain cut-off wavelength and beyond in contrast with Mie-resonance based dielectric nanostructures and metallic nanostructures whose resonance bandwidth is narrower. By the combination of constituent materials and structural parameters, HMMs can be designed to control PL in terms of enhancement, emission directivity, and statistics (single-photon emission, classical light, lasing) at any desired wavelength range in visible and near-infrared wavelength regions. Major building blocks of HMMs are metal-dielectric multilayer and metal nanowire structures that give two different types of hyperbolic region. Apart from these, more advanced structures with grating out-coupler embedded in HMM and cavity structures made out of HMM have been developed. While fabrication of HMM structures for visible to near-infrared wavelengths have been well-established, the control of light emission by HMMs can also be extended for other wavelength regime since HMM can be tailored for such longer wavelength region as mid-infrared wavelengths $s^{45,55,100,166}$. Moreover, $2 \mathrm{D}$ materials with hyperbolic dispersion for certain mid-infrared wavelengths are also shown to support directional high- $\mathrm{k}$ surface waves, including patterned hexagonal boron nitride $(\mathrm{h}-\mathrm{BN})^{167}$, $\alpha$-molybdenum trioxide $\left(\alpha-\mathrm{MoO}_{3}\right)^{168,169}$. Moreover, recently numerous natural hyperbolic materials have been proposed ${ }^{35-37}$ and discovered ${ }^{170,171}$. Especially, some of these natural materials exhibit hyperbolic dispersion from ultraviolet to near-infrared wavelengths where demand for PL control is enormous. Although their hyperbolic regions are fixed to certain bandwidth defined by materials, they do not require advanced nanofabrication technology and therefore such natural material facilitate the exploitation of hyperbolic dispersion. In this regards, two potential direction can be envisaged: exploration and characterization of such new natural hyperbolic materials and the demonstration of PL enhancement and control, as well as their applications. We believe that HMM-based systems can serve as a robust platform for PL controls in vast wavelengths regions, thus leading to numerous applications, from light sources to bioimaging and sensing. Note another important field of active HMMs application in the on-chip quantum technologies which is currently underway ${ }^{172}$.

\section{References}

1. Schirhagl R, Chang K, Loretz M, Degen CL. Nitrogen-vacancy centers in diamond: nanoscale sensors for physics and biology. Annu Rev Phys Chem 65, 83-105 (2014). 
2. Kinkhabwala A, Yu ZF, Fan SH, Avlasevich $\mathrm{Y}$, Müllen $\mathrm{K}$ et al. Large single-molecule fluorescence enhancements produced by a bowtie nanoantenna. Nat Photonics 3, 654-657 (2009).

3. Bauch M, Toma K, Toma M, Zhang QW, Dostalek J. Plasmonenhanced fluorescence biosensors: a review. Plasmonics $\mathbf{9}$, 781-799 (2014).

4. Li JF, Li CY, Aroca RF. Plasmon-enhanced fluorescence spectroscopy. Chem Soc Rev 46, 3962-3979 (2017).

5. Jeong Y, Kook YM, Lee K, Koh WG. Metal enhanced fluorescence (MEF) for biosensors: general approaches and a review of recent developments. Biosens Bioelectron 111, 102-116 (2018).

6. Zhang SL, Liu LW, Ren S, Li ZL, Zhao YH et al. Recent advances in nonlinear optics for bio-imaging applications. OptoElectron Adv 3, 200003 (2020).

7. Sultangaziyev A, Bukasov R. Review: applications of surfaceenhanced fluorescence (SEF) spectroscopy in bio-detection and biosensing. Sens Bio-Sens Res 30, 100382 (2020).

8. Joyce C, Fothergill SM, Xie F. Recent advances in gold-based metal enhanced fluorescence platforms for diagnosis and imaging in the near-infrared. Mater Today Adv 7, 100073 (2020).

9. Zhang CJ, Zhang CY, Zhang ZL, He T, Mi XH et al. Self-suspended rare-earth doped up-conversion luminescent waveguide: propa-gating and directional radiation. Opto-Electron Adv 3, 190045 (2020).

10. Ui Lee Y, Posner C, Zhao JX, Zhang J, Liu ZW. Imaging of cell morphology changes via metamaterial-assisted photobleaching microscopy. Nano Lett 21, 1716-1721 (2021).

11. Heo M, Cho H, Jung JW, Jeong JR, Park $S$ et al. High-performance organic optoelectronic devices enhanced by surface plasmon resonance. Adv Mater 23, 5689-5693 (2011).

12. Kochuveedu ST, Kim DH. Surface plasmon resonance mediated photoluminescence properties of nanostructured multicomponent fluorophore systems. Nanoscale 6, 4966-4984 (2014).

13. Chien FC, Lin CY, Abrigo G. Enhancing the blinking fluorescence of single-molecule localization imaging by using a surface-plasmon-polariton-enhanced substrate. Phys Chem Chem Phys 20, 27245-27255 (2018).

14. Curto AG, Volpe G, Taminiau TH, Kreuzer MP, Quidant R et al. Unidirectional emission of a quantum dot coupled to a nanoantenna. Science 329, 930-933 (2010).

15. Taminiau TH, Stefani FD, van Hulst NF. Enhanced directional excitation and emission of single emitters by a nano-optical Yagi-Uda antenna. Opt Express 6, 10858-10866 (2008).

16. Badshah MA, Koh NY, Zia AW, Abbas N, Zahra Z et al. Recent developments in plasmonic nanostructures for metal enhanced fluorescence-based biosensing. Nanomaterials 10, 1749 (2020).

17. Miranda B, Chu KY, Maffettone PL, Shen AQ, Funari R. Metalenhanced fluorescence immunosensor based on plasmonic arrays of gold nanoislands on an etched glass substrate. ACS Appl Nano Mater 3, 10470-10478 (2020).

18. Stella U, Boarino L, De Leo N, Munzert P, Descrovi E. Enhanced directional light emission assisted by resonant bloch surface waves in circular cavities. ACS Photonics 6, 2073-2082 (2019).

19. Toma K, Descrovi E, Toma M, Ballarini M, Mandracci P et al. Bloch surface wave-enhanced fluorescence biosensor. Biosens Bioelectron 43, 108-114 (2013).
20. Prusakov KA, Bagrov DV, Basmanov DV, Romanov SA, Klinov DV. Fluorescence imaging of cells using long-range electromagnetic surface waves for excitation. Appl Opt 59, 4833-4838 (2020).

21. Pokhriyal A, Lu M, Huang CS, Schulz S, Cunningham BT. Multicolor fluorescence enhancement from a photonics crystal surface. Appl Phys Lett 97, 121108 (2010).

22. Pokhriyal A, Lu M, Chaudhery V, George S, Cunningham BT. Enhanced fluorescence emission using a photonic crystal coupled to an optical cavity. Appl Phys Lett 102, 221114 (2013).

23. Pokhriyal A, Lu M, Chaudhery V, Huang CS, Schulz S et al. Photonic crystal enhanced fluorescence using a quartz substrate to reduce limits of detection. In CLEO: 2011 - Laser Applications to Photonic Applications CThQ1 (Optical Society of America, 2011); http://doi.org/10.1364/cleo_si.2011.cthq1.

24. Chen W, Long KD, Yu H, Tan YF, Choi JS et al. Enhanced live cell imaging via photonic crystal enhanced fluorescence microscopy. Analyst 139, 5954-5963 (2014).

25. Menon SHG, Lal Krishna AS, Raghunathan V. Silicon nitride based medium contrast gratings for doubly resonant fluorescence enhancement. IEEE Photonics J 11, 4500711 (2019).

26. Boonruang S, Srisuai N, Charlermroj R, Makornwattana M, Somboonkaew $\mathrm{A}$ et al. Excitation of multi-order guided mode resonance for multiple color fluorescence enhancement. Opt Laser Technol 106, 410-416 (2018).

27. Lin JH, Liou HY, Wang CD, Tseng CY, Lee CT et al. Giant enhancement of upconversion fluorescence of $\mathrm{NaYF}_{4}: \mathrm{Yb}^{3+}, \mathrm{Tm}^{3+}$ nanocrystals with resonant waveguide grating substrate. ACS Photonics 2, 530-536 (2015).

28. Hoang NV, Pereira A, Nguyen HS, Drouard E, Moine B et al. Giant enhancement of luminescence down-shifting by a doubly resonant rare-earth-doped photonic metastructure. ACS Photonics 4, 1705-1712 (2017).

29. Sun S, Wu L, Bai P, Png CE. Fluorescence enhancement in visible light: dielectric or noble metal? Phys Chem Chem Phys 18, 19324-19335 (2016).

30. Bucher T, Vaskin A, Mupparapu R, Löchner FJF; George A et al. Tailoring photoluminescence from $\mathrm{MoS}_{2}$ monolayers by mie-resonant metasurfaces. ACS Photonics 6, 1002-1009 (2019).

31. Lin HJ, de Oliveira Lima K, Gredin P, Mortier M, Billot L et al. Fluorescence enhancement near single $\mathrm{TiO}_{2}$ nanodisks. Appl Phys Lett 111, 251109 (2017).

32. Cortes CL, Newman W, Molesky S, Jacob Z. Quantum nanophotonics using hyperbolic metamaterials. J Opt 14, 063001 (2012).

33. Shekhar P, Atkinson J, Jacob Z. Hyperbolic metamaterials: fundamentals and applications. Nano Converg 1, 14 (2014).

34. Takayama O, Lavrinenko AV. Optics with hyperbolic materials [Invited]. J Opt Soc Am B 36, F38-F48 (2019).

35. Sun JB, Litchinitser NM, Zhou J. Indefinite by nature: from ultraviolet to terahertz. ACS Photonics 1, 293-303 (2014).

36. Korzeb K, Gajc M, Pawlak DA. Compendium of natural hyperbolic materials. Opt Express 23, 25406-25424 (2015).

37. Narimanov EE, Kildishev AV. Naturally hyperbolic. Nat Photonics 9, 214-216 (2015).

38. Ferrari L, Wu C, Lepage D, Zhang X, Liu ZW. Hyperbolic metamaterials and their applications. Prog Quantum Electron 40, 1-40 (2015). 
39. Lu L, Simpson RE, Valiyaveedu SK. Active hyperbolic metamaterials: progress, materials and design. J Opt 20, 103001 (2018).

40. Smalley JST, Vallini F, Zhang X, Fainman Y. Dynamically tunable and active hyperbolic metamaterials. Adv Opt Photonics 10, 354-408 (2018).

41. Adams DC, Inampudi S, Ribaudo T, Slocum D, Vangala S et al. Funneling light through a subwavelength aperture with epsiIon-near-zero materials. Phys Rev Lett 107, 133901 (2011).

42. Poddubny A, lorsh I, Belov P, Kivshar Y. Hyperbolic metamaterials. Nat Photonics 7, 948-957 (2013).

43. Kabashin AV, Evans P, Pastkovsky S, Hendren W, Wurtz GA et al. Plasmonic nanorod metamaterials for biosensing. Nat Mater 8, 867-871 (2009).

44. Sreekanth KV, Alapan Y, Elkabbash M, Ilker E, Hinczewski M et al. Extreme sensitivity biosensing platform based on hyperbolic metamaterials. Nat Mater 15, 621-627 (2016).

45. Shkondin E, Repän T, Panah MEA, Lavrinenko AV, Takayama O. High aspect ratio plasmonic nanotrench structures with large active surface area for label-free mid-infrared molecular absorption sensing. ACS Appl Nano Mater 1, 1212-1218 (2018).

46. Lu D, Liu ZW. Hyperlenses and metalenses for far-field superresolution imaging. Nat Commun 3, 1205 (2012).

47. Jacob Z, Narimanov EE. Optical hyperspace for plasmons: dyakonov states in metamaterials. Appl Phys Lett 93, 221109 (2008).

48. Kildishev AV, Boltasseva A, Shalaev VM. Planar photonics with metasurfaces. Science 339, 1232009 (2013).

49. Poddubny AN, Belov PA, Kivshar YS. Spontaneous radiation of a finite-size dipole emitter in hyperbolic media. Phys Rev $A$ 84, 023807 (2011).

50. Kidwai O, Zhukovsky SV, Sipe JE. Dipole radiation near hyperbolic metamaterials: applicability of effective-medium approximation. Opt Lett 36, 2530-2532 (2011).

51. Mahmoodi M, Tavassoli SH, Takayama O, Sukham J, Malureanu $\mathrm{R}$ et al. Existence conditions of high-k modes in finite hyperbolic metamaterials. Laser Photonics Rev 13, 1800253 (2019).

52. Kidwai O, Zhukovsky SV, Sipe JE. Effective-medium approach to planar multilayer hyperbolic metamaterials: strengths and limitations. Phys Rev A 85, 053842 (2012).

53. Sukham J, Takayama O, Mahmoodi M, Sychev S, Bogdanov A et al. Investigation of effective media applicability for ultrathin multilayer structures. Nanoscale 11, 12582-12588 (2019).

54. Krishnamoorthy HNS, Jacob Z, Narimanov E, Kretzschmar I, Menon VM. Topological transitions in metamaterials. Science 336, 205-209 (2012).

55. Takayama O, Shkondin E, Bodganov A, Panah MEA, Golenitskii $\mathrm{K}$ et al. Midinfrared surface waves on a high aspect ratio nanotrench platform. ACS Photonics 4, 2899-2907 (2017).

56. Smalley JST, Vallini F, Montoya SA, Ferrari L, Shahin S et al. Luminescent hyperbolic metasurfaces. Nat Commun 8, 13793 (2017).

57. Vasilantonakis N, Nasir ME, Dickson W, Wurtz GA, Zayats AV. Bulk plasmon-polaritons in hyperbolic nanorod metamaterial waveguides. Laser Photonics Rev 9, 345-353 (2015).

58. Avrutsky I, Salakhutdinov I, Elser J, Podolskiy V. Highly confined optical modes in nanoscale metal-dielectric multilayers. Phys Rev B 75, 241402 (2007).
59. Zhukovsky SV, Orlov AA, Babicheva VE, Lavrinenko AV, Sipe JE. Photonic-band-gap engineering for volume plasmon polaritons in multiscale multilayer hyperbolic metamaterials. Phys Rev A 90, 013801 (2014).

60. Takayama O, Bogdanov AA, Lavrinenko AV. Photonic surface waves on metamaterial interfaces. J Phys Condens Matter 29, 463001 (2017).

61. Higuchi M, Takahara J. Plasmonic interpretation of bulk propagating waves in hyperbolic metamaterial optical waveguides. Opt Express 26, 1918-1929 (2018).

62. Zhukovsky SV, Kidwai O, Sipe JE. Physical nature of volume plasmon polaritons in hyperbolic metamaterials. Opt Express 21, 14982-14987 (2013).

63. Poddubny AN, Belov PA, Ginzburg P, Zayats AV, Kivshar YS. Microscopic model of purcell enhancement in hyperbolic metamaterials. Phys Rev B 86, 035148 (2012).

64. Slobozhanyuk AP, Ginzburg P, Powell DA, lorsh I, Shalin AS et al. Purcell effect in hyperbolic metamaterial resonators. Phys Rev B 92, 195127 (2015).

65. Rytov SM. Electromagnetic properties of a finely stratified medium. Sov Phys JETP 2, 466-475 (1956).

66. Agranovich VM. Dielectric permeability and influence of external fields on optical properties of superlattices. Solid State Commun 78, 747-750 (1991).

67. Jacob Z, Kim JY, Naik GV, Boltasseva A, Narimanov EE et al. Engineering photonic density of states using metamaterials. Appl Phys B 100, 215-218 (2010).

68. Tumkur T, Zhu G, Black P, Barnakov YA, Bonner CE et al. Control of spontaneous emission in a volume of functionalized hyperbolic metamaterial. Appl Phys Lett 99, 151115 (2011).

69. Kim J, Drachev VP, Jacob Z, Naik GV, Boltasseva A et al. Improving the radiative decay rate for dye molecules with hyperbolic metamaterials. Opt Express 20, 8100-8116 (2012).

70. Sreekanth KV, Biaglow T, Strangi G. Directional spontaneous emission enhancement in hyperbolic metamaterials. J Appl Phys 114, 134306 (2013).

71. Shalaginov MY, Ishii S, Liu J, Liu J, Irudayaraj J et al. Broadband enhancement of spontaneous emission from nitrogen-vacancy centers in nanodiamonds by hyperbolic metamaterials. Appl Phys Lett 102, 173114 (2013).

72. Shalaginov MY, Vorobyov VV, Liu J, Ferrera M, Akimov AV et al. Enhancement of single-photon emission from nitrogen-vacancy centers with $\mathrm{TiN} /(\mathrm{Al}, \mathrm{Sc}) \mathrm{N}$ hyperbolic metamaterial. Laser Photonics Rev 9, 120-127 (2015).

73. Wang $Y$, Sugimoto $H$, Inampudi $S$, Capretti $A$, Fujii $M$ et al. Broadband enhancement of local density of states using silicon-compatible hyperbolic metamaterials. Appl Phys Lett 106, 241105 (2015).

74. Ozel T, Nizamoglu S, Sefunc MA, Samarskaya O, Ozel IO et al. Anisotropic emission from multilayered plasmon resonator nanocomposites of isotropic semiconductor quantum dots. ACS Nano 5, 1328-1334 (2011).

75. Zhukovsky SV, Ozel T, Mutlugun E, Gaponik N, Eychmuller A et al. Hyperbolic metamaterials based on quantum-dot plasmon-resonator nanocomposites. Opt Express 22, 18290-18298 (2014).

76. Lin HI, Shen KC, Liao YM, Li YH, Perumal P et al. Integration of nanoscale light emitters and hyperbolic metamaterials: an efficient platform for the enhancement of random laser action. ACS Photonics 5, 718-727 (2018). 
77. Shen KC, Hsieh C, Cheng YJ, Tsai DP. Giant enhancement of emission efficiency and light directivity by using hyperbolic metacavity on deep-ultraviolet AIGaN emitter. Nano Energy 45, 353-358 (2018).

78. Kitur JK, Gu L, Tumkur T, Bonner C, Noginov MA. Stimulated emission of surface plasmons on top of metamaterials with hyperbolic dispersion. ACS Photonics 2, 1019-1024 (2015).

79. Shen YF, Yan YX, Brigeman AN, Kim H, Giebink NC. Efficient upper-excited state fluorescence in an organic hyperbolic metamaterial. Nano Lett 18, 1693-1698 (2018).

80. Ui Lee Y, Gaudin OPM, Lee KJ, Choi E, Placide V et al. Organic monolithic natural hyperbolic material. ACS Photonics $\mathbf{6}$, 1681-1689 (2019).

81. Zhukovsky SV, Andryieuski A, Takayama O, Shkondin E, Malureanu $\mathrm{R}$ et al. Experimental demonstration of effective medium approximation breakdown in deeply subwavelength all-dielectric multilayers. Phys Rev Lett 115, 177402 (2015).

82. Mahmoodi M, Tavassoli SH, Lavrinenko AV. Mode-resolved directional enhancement of spontaneous emission inside/outside finite multilayer hyperbolic metamaterials. Mater Today Commun 23, 100859 (2020).

83. Kannegulla A, Wang YC, Liu Y, Wu B, Cheng LJ. Large-area outcoupling of quantum dot emission on multilayer hyperbolic metamaterials. In Proceedings of SPIE 10719, Metamaterials, Metadevices, and Metasystems 2018 107192K (SPIE, 2018); http://doi.org/10.1117/12.2322085.

84. Li L, Zhou ZY, Min CJ, Yuan XC. Few-layer metamaterials for spontaneous emission enhancement. Opt Lett 46, 190-193 (2021).

85. Wang L, Li SL, Zhang BR, Qin YZ, Tian Z et al. Asymmetrically curved hyperbolic metamaterial structure with gradient thicknesses for enhanced directional spontaneous emission. ACS Appl Mater Interfaces 10, 7704-7708 (2018).

86. Lin HI, Shen KC, Lin SY, Haider G, Li YH et al. Transient and flexible hyperbolic metamaterials on freeform surfaces. Sci Rep 8, 9469 (2018).

87. Inam FA, Ahmed N, Steel MJ, Castelletto S. Hyperbolic metamaterial resonator-antenna scheme for large, broadband emission enhancement and single-photon collection. $J \mathrm{Opt}$ Soc Am B 35, 2153-2162 (2018).

88. Kala A, Inam FA, Biehs SA, Vaity P, Achanta VG. Hyperbolic metamaterial with quantum dots for enhanced emission and collection efficiencies. Adv Opt Mater 8, 2000368 (2020).

89. Yang XD, Yao J, Rho J, Yin XB, Zhang X. Experimental realization of three-dimensional indefinite cavities at the nanoscale with anomalous scaling laws. Nat Photonics 6, 450-454 (2012).

90. Indukuri SRKC, Frydendahl C, Bar-David J, Mazurski N, Levy $\mathrm{U}$. $\mathrm{WS}_{2}$ monolayers coupled to hyperbolic metamaterial nanoantennas: broad implications for light-matter-interaction applications. ACS Appl Nano Mater 3, 10226-10233 (2020).

91. Forster T. Energiewanderung und fluoreszenz. Naturwissenschaften 33, 166-175 (1946).

92. Förster T. Zwischenmolekulare energiewanderung und fluoreszenz. Ann Phys 437, 55-75 (1948).

93. Tumkur TU, Kitur JK, Bonner CE, Poddubny AN, Narimanov $E E$ et al. Control of Förster energy transfer in the vicinity of metallic surfaces and hyperbolic metamaterials. Faraday Discuss 178, 395-412 (2015)

94. Adl HP, Gorji S, Habil MK, Suárez I, Chirvony VS et al. Purcell enhancement and wavelength shift of emitted light by $\mathrm{CsPbl}_{3}$ perovskite nanocrystals coupled to hyperbolic metamaterials. ACS Photonics 7, 3152-3160 (2020).

95. Tonkaev P, Anoshkin S, Pushkarev A, Malureanu R, Masharin $M$ et al. Acceleration of radiative recombination in quasi-2D perovskite films on hyperbolic metamaterials. Appl Phys Lett 118, 091104 (2021)

96. Lin HI, Wang CC, Shen KC, Shalaginov MY, Roy PK et al. Enhanced laser action from smart fabrics made with rollable hyperbolic metamaterials. npj Flex Electron 4, 20 (2020).

97. Moritake Y, Nakayama K, Suzuki T, Kurosawa H, Kodama T et al. Lifetime reduction of a quantum emitter with quasiperiodic metamaterials. Phys Rev B 90, 075146 (2014).

98. Li L, Mathai CJ, Gangopadhyay S, Yang XD, Gao J. Spontaneous emission rate enhancement with aperiodic Thue-Morse multilayer. Sci Rep 9, 8473 (2019).

99. High AA, Devlin RC, Dibos A, Polking M, Wild DS et al. Visiblefrequency hyperbolic metasurface. Nature 522, 192-196 (2015).

100. Takayama O, Dmitriev P, Shkondin E, Yermakov O, Panah M et al. Experimental observation of dyakonov plasmons in the mid-infrared. Semiconductors 52, 442-446 (2018).

101. Li ZT, Smalley JST, Haroldson R, Lin DY, Hawkins R et al. Active perovskite hyperbolic metasurface. ACS Photonics 7, 1754-1761 (2020).

102. Noginov MA, Li H, Barnakov YA, Dryden D, Nataraj G et al. Controlling spontaneous emission with metamaterials. Opt Lett 35, 1863-1865 (2010).

103. Roth DJ, Krasavin AV, Wade A, Dickson W, Murphy A et al. Spontaneous emission inside a hyperbolic metamaterial waveguide. ACS Photonics 4, 2513-2521 (2017).

104. Ginzburg P, Roth DJ, Nasir ME, Segovia P, Krasavin AV et al. Spontaneous emission in non-local materials. Light: Sci Appl 6, e16273 (2017).

105. Roth DJ, Nasir ME, Ginzburg P, Wang P, Le Marois A et al. Förster resonance energy transfer inside hyperbolic metamaterials. ACS Photonics 5, 4594-4603 (2018).

106. Córdova-Castro RM, Krasavin AV, Nasir ME, Zayats AV, Dickson W. Nanocone-based plasmonic metamaterials. Nanotechnology 30, 055301 (2019).

107. Roth DJ, Ginzburg P, Hirvonen LM, Levitt JA, Nasir ME et al. Singlet-triplet transition rate enhancement inside hyperbolic metamaterials. Laser Photonics Rev 13, 1900101 (2019).

108. Chandrasekar R, Wang ZX, Meng XG, Azzam SI, Shalaginov $\mathrm{MY}$ et al. Lasing action with gold nanorod hyperbolic metamaterials. ACS Photonics 4, 674-680 (2017).

109. Indukuri C, Yadav RK, Basu JK. Broadband room temperature strong coupling between quantum dots and metamaterials. Nanoscale 9, 11418-11423 (2017).

110. Lu D, Kan JJ, Fullerton EE, Liu ZW. Enhancing spontaneous emission rates of molecules using nanopatterned multilayer hyperbolic metamaterials. Nat Nanotechno/ 9, 48-53 (2014).

111. Lu D, Ferrari L, Kan JJ, Fullerton EE, Liu ZW. Optimization of nanopatterned multilayer hyperbolic metamaterials for spontaneous light emission enhancement. Phys Status Solidi (A) 215, 1800263 (2018).

112. Li L, Wang W, Luk TS, Yang XD, Gao J. Enhanced quantum dot spontaneous emission with multilayer metamaterial nanostructures. ACS Photonics 4, 501-508 (2017).

113. Sreekanth KV, Krishna KH, De Luca A, Strangi G. Large 
spontaneous emission rate enhancement in grating coupled hyperbolic metamaterials. Sci Rep 4, 6340 (2014).

114. Galfsky T, Gu J, Narimanov EE, Menon VM. Photonic hypercrystals for control of light-matter interactions. Proc Natl Acad Sci USA 114, 5125-5129 (2017).

115. Gan QQ, Gao YK, Wagner K, Vezenov D, Ding YJ et al. Experimental verification of the rainbow trapping effect in adiabatic plasmonic gratings. Proc Natl Acad Sci USA 108, 5169-5173 (2011).

116. Ni X, Wu Y, Chen ZG, Zheng LY, Xu YL et al. Acoustic rainbow trapping by coiling up space. Sci Rep 4, 7038 (2014).

117. Hu HF, Ji DX, Zeng X, Liu K, Gan QQ. Rainbow trapping in hyperbolic metamaterial waveguide. Sci Rep 3, 1249 (2013).

118. Zhou J, Kaplan AF, Chen L, Guo LJ. Experiment and theory of the broadband absorption by a tapered hyperbolic metamaterial array. ACS Photonics 1, 618-624 (2014).

119. Krishna KH, Sreekanth KV, Strangi G. Dye-embedded and nanopatterned hyperbolic metamaterials for spontaneous emission rate enhancement. J Opt Soc Am B 33, 1038-1043 (2016).

120. Galfsky T, Sun Z, Considine CR, Chou CT, Ko WC et al. Broadband enhancement of spontaneous emission in two-dimensional semiconductors using photonic hypercrystals. Nano Lett 16, 4940-4945 (2016).

121. Newman WD, Cortes CL, Jacob Z. Enhanced and directional single-photon emission in hyperbolic metamaterials. J Opt Soc Am B 30, 766-775 (2013).

122. Galfsky T, Krishnamoorthy HNS, Newman W, Narimanov EE, Jacob $Z$ et al. Active hyperbolic metamaterials: enhanced spontaneous emission and light extraction. Optica 2, 62-65 (2015).

123. Cheng Y, Liao CT, Xie ZH, Hung YC, Lee MC. Study of cavityenhanced dipole emission on a hyperbolic metamaterial slab. $J$ Opt Soc Am B 36, 426-434 (2019).

124. Niu XX, Hu XY, Chu SS, Gong QH. Epsilon-near-zero photonics: a new platform for integrated devices. Adv Opt Mater 6, 1701292 (2018).

125. Vulis DI, Reshef O, Camayd-Muñoz P, Mazur E. Manipulating the flow of light using Dirac-cone zero-index metamaterials. Rep Prog Phys 82, 012001 (2019).

126. Campione S, Brener I, Marquier F. Theory of epsilon-near-zero modes in ultrathin films. Phys Rev B 91, 121408 (2015).

127. Nordin L, Dominguez O, Roberts CM, Streyer W, Feng K et al. Mid-infrared epsilon-near-zero modes in ultra-thin phononic films. Appl Phys Lett 111, 091105 (2017).

128. Folland TG, Lu GY, Bruncz A, Nolen JR, Tadjer M et al. Vibrational coupling to epsilon-near-zero waveguide modes. ACS Photonics 7, 614-621 (2020).

129. Engheta N. Pursuing near-zero response. Science $\mathbf{3 4 0}$, 286-287 (2013).

130. Javani MH, Stockman MI. Real and imaginary properties of epsilon-near-zero materials. Phys Rev Lett 117, 107404 (2106).

131. Fleury R, Alù $A$. Enhanced superradiance in epsilon-near-zero plasmonic channels. Phys Rev B 87, 201101 (2013).

132. Sreekanth KV, De Luca A, Strangi G. Experimental demonstration of surface and bulk plasmon polaritons in hypergratings. Sci Rep 3, 3291 (2013).

133. Caligiuri V, Dhama R, Sreekanth KV, Strangi G, De Luca A. Dielectric singularity in hyperbolic metamaterials: the inversion point of coexisting anisotropies. Sci Rep 6, 20002 (2016).
134. Drachev VP, Podolskiy VA, Kildishev AV. Hyperbolic metamaterials: new physics behind a classical problem. Opt Express 21, 15048-15064 (2013).

135. Caligiuri V, Palei M, Imran M, Manna L, Krahne R. Planar double-epsilon-near-zero cavities for spontaneous emission and purcell effect enhancement. ACS Photonics 5, 2287-2294 (2018).

136. Walt DR. Optical methods for single molecule detection and analysis. Anal Chem 85, 1258-1263 (2013).

137. Melentiev PN, Son LV, Kudryavtsev DS, Kasheverov IE, Tsetlin $\mathrm{VI}$ et al. Ultrafast, ultrasensitive detection and imaging of single cardiac troponin-T molecules. ACS Sens 5, 3576-3583 (2020).

138. Engheta N. Circuits with light at nanoscales: optical nanocircuits inspired by metamaterials. Science 317, 1698-1702 (2007).

139. Melentiev PN, Kalmykov A, Kuzin A, Negrov D, Klimov V et al. Open-type SPP waveguide with ultrahigh bandwidth up to 3.5 THz. ACS Photonics 6, 1425-1433 (2019).

140. Argyropoulos C, Estakhri NM, Monticone F, Alù A. Negative refraction, gain and nonlinear effects in hyperbolic metamaterials. Opt Express 21, 15037-15047 (2013).

141. Wan MJ, Gu P, Liu WY, Chen Z, Wang ZL. Low threshold spaser based on deep-subwavelength spherical hyperbolic metamaterial cavities. Appl Phys Lett 110, 031103 (2017).

142. Janaszek B, Tyszka-Zawadzka A, Szczepański P. Control of gain/absorption in tunable hyperbolic metamaterials. Opt Express 25, 13153-13162 (2017).

143. Bergman DJ, Stockman MI. Surface plasmon amplification by stimulated emission of radiation: quantum generation of coherent surface plasmons in nanosystems. Phys Rev Lett 90, 027402 (2003).

144. Bozhevolnyi SI, Khurgin JB. The case for quantum plasmonics. Nat Photonics 11, 398-400 (2017).

145. del Valle E, Laussy FP, Tejedor C. Luminescence spectra of quantum dots in microcavities. Phys $\operatorname{Rev} B, 79,235326$ (2009).

146. Shahbazyan TV. Exciton-plasmon energy exchange drives the transition to a strong coupling regime. Nano Lett 19 3273-3279 (2019).

147. Khurgin JB. Exceptional points in polaritonic cavities and subthreshold fabry-perot lasers. Optica 7, 1015-1023 (2020).

148. Pustovit VN, Urbas AM, Zelmon DE. Surface plasmon amplification by stimulated emission of radiation in hyperbolic metamaterials. Phys Rev B 94, 235445 (2016).

149. Vaianella F, Hamm JM, Hess O, Maes B. Strong coupling and exceptional points in optically pumped active hyperbolic metamaterials. ACS Photonics 5, 2486-2495 (2018).

150. Ni X, Naik GV, Kildishev AV, Barnakov Y, Boltasseva A et al. Effect of metallic and hyperbolic metamaterial surfaces on electric and magnetic dipole emission transitions. Appl Phys $B$ 103, 553-558 (2011).

151. Michler P, Kiraz A, Becher C, Schoenfeld WV, Petroff PM et al. A quantum dot single-photon turnstile device. Science $\mathbf{2 9 0}$ 2282-2285 (2000).

152. Pelton M, Santori C, Vučković J, Zhang BY, Solomon GS et al. Efficient source of single photons: a single quantum dot in a micropost microcavity. Phys Rev Lett 89, 233602 (2002).

153. Lodahl P, van Driel AF, Nikolaev IS, Irman A, Overgaag $\mathrm{K}$ et al. Controlling the dynamics of spontaneous emission from 
quantum dots by photonic crystals. Nature 430, 654-657 (2004).

154. Khitrova G, Gibbs HM, Kira M, Koch SW, Scherer A. Vacuum Rabi splitting in semiconductors. Nat Phys 2, 81-90 (2006).

155. Rogacheva AV, Fedotov VA, Schwanecke AS, Zheludev NI. Giant gyrotropy due to electromagnetic-field coupling in a bilayered chiral structure. Phys Rev Lett 97, 177401 (2006).

156. Ameling R, Dregely D, Giessen H. Strong coupling of localized and surface plasmons to microcavity modes. Opt Lett $\mathbf{3 6}$, 2218-2220 (2011).

157. Goldzak T, Mailybaev AA, Moiseyev N. Light stops at exceptional points. Phys Rev Lett 120, 013901 (2018).

158. Pick A, Zhen B, Miller OD, Hsu CW, Hernandez F et al. General theory of spontaneous emission near exceptional points. Opt Express 25, 12325-12348 (2017).

159. Hou JP, Li ZT, Luo XW, Gu Q, Zhang CW. Topological bands and triply degenerate points in non-hermitian hyperbolic metamaterials. Phys Rev Lett 124, 073603 (2020).

160. Doronin IV, Zyablovsky AA, Andrianov ES. Strong-coupling-assisted formation of coherent radiation below the lasing threshold. arXiv: 2012.05288 (2020).

161. Antosiewicz TJ, Apell SP, Shegai T. Plasmon-exciton interactions in a core-shell geometry: from enhanced absorption to strong coupling. ACS Photonics 1, 454-463 (2014).

162. Shen KC, Ku CT, Hsieh C, Kuo HC, Cheng YJ et al. Deep-ultraviolet hyperbolic metacavity laser. Adv Mater 30, 1706918 (2018).

163. Shishkov VY, Andrianov ES, Pukhov AA, Vinogradov AP. Enhancement of nonclassical raman light intensity by plasmonic nanoantenna. Phys Rev A 103, 013725 (2021).

164. del Pino J, Feist J, Garcia-Vidal FJ. Signatures of vibrational strong coupling in raman scattering. J. Phys Chem C 119, 29132-29137 (2015).

165. Waks E, Sridharan D. Cavity QED treatment of interactions between a metal nanoparticle and a dipole emitter. Phys Rev A 84, 043845 (2010).

166. Hoffman AJ, Alekseyev L, Howard SS, Franz KJ, Wasserman
$D$ et al. Negative refraction in semiconductor metamaterials. Nat Mater 6, 946-950 (2007).

167. Li PN, Dolado I, Alfaro-Mozaz FJ, Casanova F, Hueso LE et al. Infrared hyperbolic metasurface based on nanostructured van der Waals materials. Science 359, 892-896 (2018).

168. Ma WL, Alonso-González P, Li SJ, Nikitin AY, Yuan J et al. Inplane anisotropic and ultra-low-loss polaritons in a natural van der Waals crystal. Nature 562, 557-562 (2018).

169. Wang C, Huang SY, Xing QX, Xie YG, Song CY et al. Van der Waals thin films of $\mathrm{WTe}_{2}$ for natural hyperbolic plasmonic surfaces. Nat Commun 11, 1158 (2020).

170. Gao H, Zhang XM, Li WF, Zhao MW. Tunable broadband hyperbolic light dispersion in metal diborides. Opt Express 27, 36911-36922 (2019).

171. Gao H, Sun L, Zhao MW. Low-loss hyperbolic dispersion and anisotropic plasmonic excitation in nodal-line semimetallic yttrium nitride. Opt Express 28, 22076-22087 (2020).

172. Kan YH, Kumar S, Ding F, Zhao CY, Bozhevolnyi SI. On-chip spin-orbit controlled excitation of quantum emitters coupled to hybrid plasmonic nanocircuits. arXiv: 2004.07483 (2020).

\section{Acknowledgements}

L. Y. Beliaev, O. Takayama and A. V. Lavrinenko acknowledge the financial support from Independent Research Fund Denmark (DFF) (Research Project 2, 8022-00387B), Denmark. P.M. acknowledges that the publication was prepared within the framework of Academic Fund Program at the HSE University in 2021 (grant No 21-04-056).

\section{Author contributions}

L. Y. Beliaev carried out the literature search. L. Y. Beliaev and O. Takayama prepared the first draft of the paper except active HMMs section. P. N. Melentiev prepared the first draft of the active HMMs section. All authors edited the manuscript and participated in discussion of the content.

\section{Competing interests}

The authors declare no competing financial interests. 